DESY 10-200

HU-EP-10/74

SFB/CPP-10-109

\title{
NGluon: A Package to Calculate One-loop Multi-gluon Amplitudes
}

\author{
S. Badger ${ }^{a}$, B. Biedermann ${ }^{b}$ and P. Uwer $^{b}$ \\ ${ }^{a}$ Deutsches Elektronensynchrotron DESY \\ Platanenallee 6, D-15735 Zeuthen, Germany \\ $\&$ \\ The Niels Bohr International Academy and Discovery Center \\ The Niels Bohr Institute \\ Blegdamsvej 17, DK-2100 Copenhagen, Denmark \\ ${ }^{b}$ Humboldt-Universität zu Berlin, Institut für Physik \\ Newtonstraße 15, D-12489 Berlin, Germany
}

\begin{abstract}
We present a computer library for the numerical evaluation of colourordered $n$-gluon amplitudes at one-loop order in pure Yang-Mills theory. The library uses the recently developed technique of generalised unitarity. Running in double precision the library yields reliable results for up to 14 gluons with only a small fraction of events requiring a re-evaluation using extended floating point arithmetic. We believe that the library presented here provides an important contribution to future LHC phenomenology. The program may also prove useful in cross checking results obtained by other methods. In addition, the code provides a sample implementation which may serve as a starting point for further developments.
\end{abstract}




\section{Program summary}

Title of program: NGluon

Version:

1.1

Catalogue number:

Program summary URL

http://www.physik.hu-berlin.de/pep/tools

E-mail:

Simon.Badger@nbi.dk, Benedikt.Biedermann@Physik.HU-Berlin.de, Peter.Uwer@Physik.HU-Berlin.de

License: $\quad$ GNU Public License

Computers:

Operating system:

Program language:

Memory required to execute:

Other programs called:

External files needed:

Keywords:

Nature of the physical problem:

Method of solution:

Restrictions on complexity of the problem:

Typical running time:
Any computer platform supported by the GNU compiler suite.

—no specific requirements_-, tested on Scientific Linux 5.2

$\mathrm{C}++$

Depending on the complexity, for realistic applications like 10 gluon production in double precision below $10 \mathrm{MB}$

-none-

QCDLoop, qd

unitarity method, one-loop corrections

Evaluation of next-to-leading order corrections for gluon scattering amplitudes in pure gauge theory.

Purely numerical approach based on tree amplitudes obtained via Berends-Giele recursion combined with unitarity method

Running in double precision the number of gluons should not exceed 14

Depending on the number of external gluons between less than a milli second ( 4 gluons) up to a 1 s (14 gluons) per phase space point. 


\section{Introduction}

The Large Hadron Collider at CERN allows the exploration of a complete new energy regime and will help us to unravel the mechanism of electroweak symmetry breaking. However, the large QCD background to essentially all major signal processes makes any potential discovery at the LHC a highly non-trivial endeavour. A necessary prerequisite is thus a solid understanding of the QCD backgrounds. This includes sophisticated methods to determine the background from data but also improved theoretical calculations providing reliable predictions. Leading-order predictions in QCD are usually plagued by large uncertainties due to the residual scale dependence. For reliable predictions higher order corrections, in particular next-to-leading order (NLO) calculations, are mandatory. With an increasing number of particles involved in the hard scattering process the evaluation of the corresponding one-loop amplitudes becomes more and more complicated. In recent years considerable progress has been made towards a fully automated procedure for NLO corrections to perturbative QCD cross sections. The virtual corrections to multi-particle amplitudes were for a long time considered to be the bottleneck in multi-jet cross sections predictions for high energy hadron collisions at the Tevatron and LHC. Over the past 15 years essentially two methods have been used. One is the traditional approach based on the evaluation of Feynman diagrams. In this approach the large number of Feynman diagrams and related to that the increasing algebraic complexity may actually put limitations on the processes which are feasible following this technique. Also numerical stability and speed are non-trivial issues. However despite these problems many important results have been obtained along these lines (see for example [1-3], we refer to Ref. [4] for a more complete review of the current status). The second method makes use of unitarity and in its original version tries to reconstruct the loop amplitudes via the Cutkosky rules. The first applications of this method to jet physics date back to the mid nineties $[5,6]$. At that time the method was used only by very few groups. This situation has changed dramatically in the past five years and the method of using unitarity cuts to construct one-loop gauge theory amplitudes is by now well established. In more recent years, thanks to a deeper understanding in the role of complex analysis, the procedure has been generalised to incorporate multiple cuts [7] effectively reducing the computation of one-loop amplitudes to an algebraic procedure where the only input from the underlying field theoretical model is provided by the respective Born amplitudes. For a detailed description of various aspects of this approach we refer to the vast literature on the subject [8-19].

In this work we follow the algorithm of $D$-dimensional generalised unitarity $[17,20]$ which is closely related to the integrand reduction of Ossola, Papadopoulos and Pittau (OPP) [8]. This procedure has been implemented successfully in a number of independent, private, codes [21-25] that have been recently applied to phenomenological NLO QCD studies (see for example Refs. [26-36]). In addition two public codes implementing the OPP integrand reduction procedure have been released $[37,38]$.

This article is organised as follows. In section 2 we give a brief overview of the on-shell techniques implemented in the NGluon $\mathrm{C}++$ package. In section 3 we describe how to install the package from the source files. A short description of the various public member functions is 
presented in section 4 . In section 5 we give some basic examples on how to use the package and show a detailed analysis on the performance in terms of speed and accuracy before reaching the conclusions in section 6 .

\section{Methods}

Since the method has been described in detail in the literature, in this section we present a basic overview of the generalised unitarity procedure focusing on the algorithm employed in NGluon. We restrict the discussion to the purely massless case throughout. Owing to the choice of the Van Neerven-Vermaseren basis for the loop momenta, our implementation most closely resembles that used by the Rocket collaboration $[17,20]$.

We split the one-loop gluon amplitudes $A_{n}^{(1)}$ into two contributions:

$$
A_{n}^{(1)}=A_{n}^{(1), c c}+R_{n}^{(1)}
$$

The cut-constructible part $A_{n}^{(1), c c}$, which contains all logarithms and divergences, may be computed using four-dimensional cuts. The remaining rational terms $R_{n}^{(1)}$ must be extracted using additional information from cuts in $4-2 \varepsilon$ dimensions. It is well known that the cutconstructible part can be written in terms of a basis of scalar integrals with a maximum of four propagators. Restricting ourselves to the case of massless propagators we write the cutconstructible term as,

$$
A_{n}^{(1), c c}=\sum_{i, j, k, l} C_{4 ; i|j| k \mid l} I_{4 ; i|j| k \mid l}+\sum_{i, j, k} C_{3 ; i|j| k} I_{3 ; i|j| k}+\sum_{i, j} C_{2 ; i \mid j} I_{2 ; i \mid j}
$$

where $I_{4}, I_{3}$ and $I_{2}$ denote the scalar four-, three- and two-point one-loop integrals. Denoting the set of external momenta as $\left\{p_{i}\right\}, i=1, n$, we label the possible internal propagators as:

$$
P_{i}=\frac{1}{D_{i}}=\frac{1}{\left(\ell-q_{i}\right)^{2}},
$$

with an integer $i$, where $q_{i}=\sum_{m=0}^{i} p_{m}$. In this notation we take $p_{0}=0$. The scalar integrals are then given by the collection of propagators as specified by the second multi-index, i.e.

$$
I_{4 ; i|j| k \mid l}=\int \frac{d^{D} \ell}{(2 \pi)^{D}} \frac{1}{D_{i} D_{j} D_{k} D_{l}} .
$$

For QCD processes these integrals are known in the framework of dimensional regularisation [39-42] and have been more recently made available in a number of public codes FF/ QCDloop [43,44], LoopTools [45] and OneLOop [46]. Therefore, the only process dependent information in Eq. (2) are the rational coefficients, $C_{4}, C_{3}$ and $C_{2}$. 
The rational part $R_{n}$ can be derived by taking the $\varepsilon \rightarrow 0$ limit of the expanded integral basis in higher dimensions [17]. After terms of higher order in $\varepsilon$ are discarded we can write,

$$
R_{n}^{(1)}=-\frac{1}{6} \sum_{i, j, k, l} C_{4 ; i|j| k \mid l}^{[4]}-\frac{1}{2} \sum_{i, j, k} C_{3 ; i|j| k}^{[2]}-\sum_{i, j} \frac{s_{i, j-1}}{6} C_{2 ; i \mid j}^{[2]}
$$

As we will discuss later the super-scripts correspond to the polynomial structure of the $D$ dimensional integrands. We choose to extract the values of these coefficients by performing the cuts in four dimensions with an internal mass carrying the $D$-dimensional information. The coefficients are then computed from the large mass limit of the four-dimensional case. This is a numerical translation of the method described in Ref. [19] which has also been used in the recent computation of $W / Z+3 j$ and $W+4 j$ by the BlackHat collaboration $[26,28,29,47]$. Alternatively one can extract the same coefficients by interpolating the result of computations in higher integer dimensions as described by Giele, Kunszt and Melnikov [17].

We follow a top-down approach, starting with the leading singularity coming from box contributions, working through the triangles to the bubbles. At each stage all possible configurations of propagators are put on-shell where the one-loop amplitude factorises into products of tree level amplitudes. Knowledge of the amplitude coming from higher cuts is then systematically removed in such a way that the integral coefficients can be uniquely identified and their value determined using purely algebraic methods.

\subsection{Universal pole structure}

The poles in the dimensional regularisation parameter, $\varepsilon$, have a universal structure which, for the case of massless QCD, has been known for some time, see for example [48]. This serves as an internal cross check for our computation and takes an extremely simple form for the colour-ordered gluon amplitudes considered in this paper,

$$
\left.A_{n}^{(1)}\left(\left\{p_{k}\right\}\right)\right|_{\text {poles }}=\left[\frac{11}{3 \varepsilon}-\frac{1}{\varepsilon^{2}} \sum_{i=1}^{n}\left(\frac{\mu_{R}^{2}}{-s_{i, i+1}}\right)^{\varepsilon}\right] A_{n}^{(0)}\left(\left\{p_{k}\right\}\right)
$$

where $s_{i, i+1}=\left(p_{i}+p_{i+1}\right)^{2}$ and $\mu_{R}$ is the regularisation scale.

\subsection{Tree-level amplitudes}

The main ingredient for the construction of the one-loop amplitude is an efficient evaluation of the tree-level amplitudes entering each cut. We have chosen to implement Berends-Giele recursion relations [49] for the gluonic tree amplitudes and the amplitudes with a pair of massive scalars relevant for the rational terms. This gives us the benefit of polynomial growth in speed with the number of gluons. The computational costs for the evaluation of pure gluon amplitudes scales like $n^{4}$ where $n$ is the number of gluons. In our implementation we cache 
all the sub-currents related to the evaluation of the Born amplitude for the process under consideration. Using this cache the evaluation of every individual sub-amplitude required for the computation of the one-loop amplitudes scales then like $n^{3}$. We will come back to this issue when we discuss the overall behaviour.

\subsection{Cut integrals and the loop momentum parametrisation}
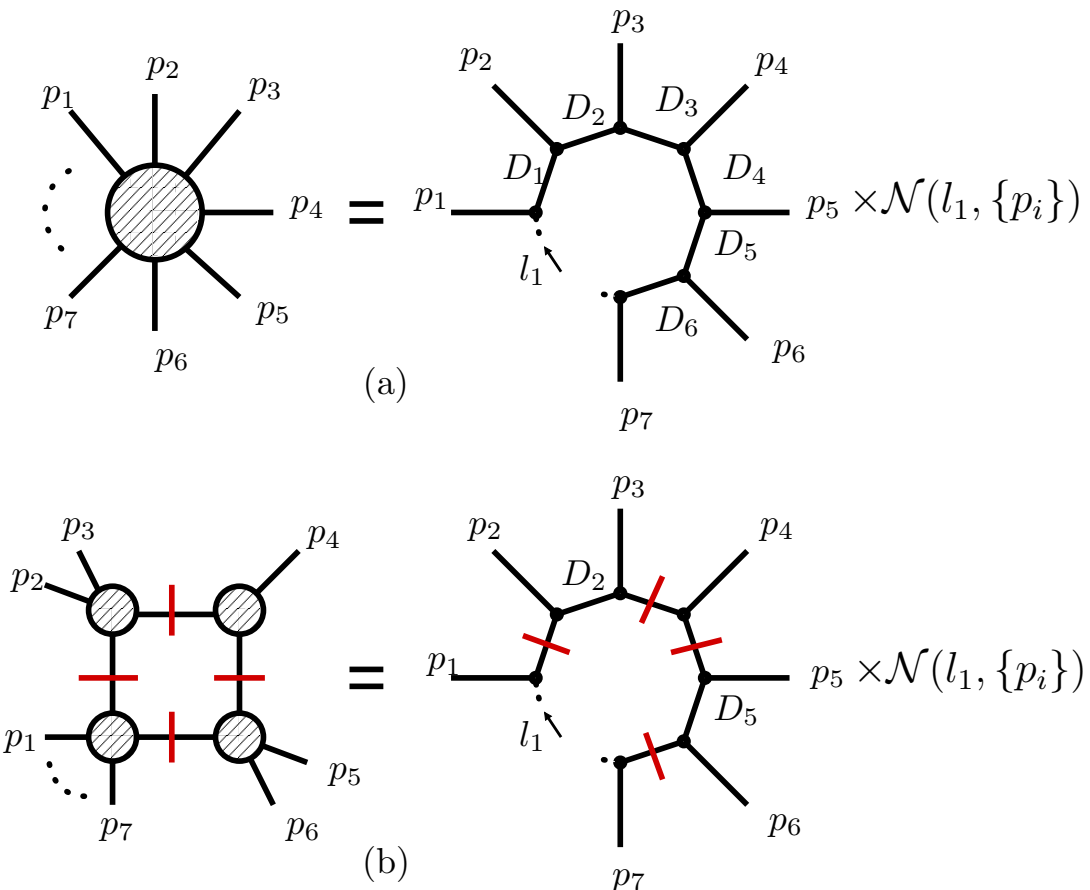

Figure 1: Figure 1(a) shows the labelling of the propagators in the integral representation of the amplitude. Figure 1(b) shows a generic quadruple cut where propagators $D_{1}, D_{3}, D_{4}$ and $D_{6}$ are replaced by on-shell delta functions and the amplitude factorises into a product of four tree amplitudes.

We choose to compute the four-dimensional complex momenta appearing as solutions to the cut constraints using the van-Neerven-Vermaseren basis [50] as described in Refs. [17,20]. An alternative approach is to use a Weyl spinor basis as used by Blackhat [21] and within the OPP approach of Ref. [51].

A complete discussion of the method has been clearly presented in Refs. [17, 20]. Here we review the essential details specific to our implementation. A general representation of the cut integrals appearing in $n$-particle one-loop processes is,

$$
\left.A_{n}^{(1)}\left(\left\{p_{i}\right\}\right)\right|_{k-c u t}=\int \frac{d^{D} \ell_{1}}{(4 \pi)^{D}}\left(\prod_{i=1}^{k} \delta\left(D_{i}\right) D_{i}\right) \frac{\mathcal{N}\left(\ell_{1},\left\{p_{i}\right\}\right)}{\prod_{j=1}^{n} D_{j}}
$$


where the propagators and external momenta are defined in eq. (3). The general loop momentum parametrisation for the $k$-particle cut in four dimensions is then written as

$$
\ell_{1 ; k}^{\mu}=V_{k}^{\mu}+\sum_{i=1}^{5-k} \alpha_{i} n_{i}^{\mu},
$$

where the unit vectors $n_{i}^{\mu}$ span the trivial space orthogonal to the physical space vector $V_{k}^{\mu}$ :

$$
V_{k} \cdot n_{j}=0, \quad n_{i} \cdot n_{j}=\delta_{i j},
$$

where $i, j=k \ldots 4$. The physical space vector $V_{k}^{\mu}$ can be determined from the external momenta $\left\{p_{i}\right\}$.

\subsection{Cut-constructible contributions}

\subsubsection{The box coefficients}

The first step is to compute the leading singularity of the one-loop amplitude coming from the quadruple cuts. We construct all possible ways to cut four propagators in the amplitude, in each case using the on-shell delta functions to freeze the loop integration. The general solution to the on-shell constraints yields two complex solutions and the final result for the box coefficient is simply the sum over the product of four trees evaluated at each solution [7]. We first denote the integrand, summed over internal helicities, as

$$
\begin{array}{r}
\bar{C}_{4 ; i|j| k \mid l}=\sum_{h_{1}, h_{2}, h_{3}, h_{4}} A^{(0)}\left(-\ell_{1}^{-h_{1}}, K_{i, j-1}, \ell_{2}^{h_{2}}\right) A^{(0)}\left(-\ell_{2}^{-h_{2}}, K_{j, k-1}, \ell_{3}^{h_{3}}\right) \\
\times A^{(0)}\left(-\ell_{3}^{-h_{3}}, K_{k, l-1}, \ell_{4}^{h_{4}}\right) A^{(0)}\left(-\ell_{4}^{-h_{4}}, K_{l, i-1}, \ell_{1}^{h_{1}}\right),
\end{array}
$$

where $K_{m, n-1}$ denote sums over external momenta defined as $K_{m, n-1}=\sum_{i=m}^{n-1} p_{i}$. The integrand has a polynomial structure depending on a vector $n_{1}$ in the trivial space satisfying $K_{i, j-1} \cdot n_{1}=$ $0, K_{j, k-1} \cdot n_{1}=0, K_{k, l-1} \cdot n_{1}=0$ and $n_{1} \cdot n_{1}=1$,

$$
\bar{C}_{4 ; i|j| k \mid l}\left(\ell_{1}\right)=C_{4 ; i|j| k \mid l}+\tilde{C}_{4 ; i|j| k \mid l} n_{1} \cdot \ell_{1}
$$

It has been shown in Ref. [20] that

$$
\begin{aligned}
& C_{4 ; i|j| k \mid l}=\frac{1}{2}\left(\bar{C}_{4 ; i|j| k \mid l}\left(\ell_{1}^{+}\right)+\bar{C}_{4 ; i|j| k \mid l}\left(\ell_{1}^{-}\right)\right) \\
& \tilde{C}_{4 ; i|j| k \mid l}=\frac{1}{2 \sqrt{\Delta_{4}}}\left(\bar{C}_{4 ; i|j| k \mid l}\left(\ell_{1}^{+}\right)-\bar{C}_{4 ; i|j| k \mid l}\left(\ell_{1}^{-}\right)\right),
\end{aligned}
$$

where the Gram determinant $\Delta_{4}$ can be written in terms of the momenta entering the propagators as,

$$
\Delta_{4}=\operatorname{det}(\mathbf{G}), \quad G_{i j}=q_{i} \cdot q_{j} .
$$

It is a convenient feature of the van Neerven-Vermaseren basis that $\Delta_{4}$ and $n_{1}$ appear naturally in the construction of the on-shell loop momenta. 


\subsubsection{The triangle coefficients}

With only three on-shell constraints, the loop integration of the triple cut has a single degree of freedom. Building upon the work of [52], it was shown in Ref. [8] that by parameterising the integrand using unit vectors $n_{1}$ and $n_{2}$ spanning the trivial space, the unknown information can be extracted as the solution to a system of linear equations. Forde then elegantly demonstrated that one can use simple complex analysis to show how the box contributions untangle from the triple cut and a subtracted integrand with polynomial behaviour leads directly to the scalar triangle coefficient [9]. Following the construction of [20], we implement this procedure by writing the integrand as,

$$
\begin{aligned}
\bar{C}_{3 ; i|j| k}= & \sum_{h_{1}, h_{2}, h_{3}} A^{(0)}\left(-\ell_{1}^{-h_{1}}, K_{i, j-1}, \ell_{2}^{h_{2}}\right) A^{(0)}\left(-\ell_{2}^{-h_{2}}, K_{j, k-1}, \ell_{3}^{h_{3}}\right) A^{(0)}\left(-\ell_{3}^{-h_{3}}, K_{k, l-1}, \ell_{1}^{h_{1}}\right) \\
& -\sum_{l} \frac{\bar{C}_{4 ; i|j| k \mid l}\left(\ell_{1}\right)}{\left(\ell_{1}-K_{l}\right)^{2}}
\end{aligned}
$$

The polynomial structure in the trivial space can then be written [20]:

$$
\begin{aligned}
\bar{C}_{3 ; i|j| k}\left(\ell_{1}\right) & =C_{3 ; i|j| k}^{(0)}+C_{3 ; i|j| k}^{(1)} \alpha_{1}+C_{3 ; i|j| k}^{(2)} \alpha_{2}+C_{3 ; i|j| k}^{(3)}\left(\alpha_{1}^{2}-\alpha_{2}^{2}\right) \\
& +C_{3 ; i|j| k}^{(4)} \alpha_{1} \alpha_{2}+C_{3 ; i|j| k}^{(5)} \alpha_{1}^{2} \alpha_{2}+C_{3 ; i|j| k}^{(6)} \alpha_{1} \alpha_{2}^{2},
\end{aligned}
$$

where $\alpha_{k}=n_{k} \cdot \ell_{1}, k=1,2$. The scalar triangle coefficient is simply $C_{3 ; i|j| k}=C_{3 ; i|j| k}^{(0)}$ We extract the coefficients $C_{3 ; i|j| k}^{(m)}$ using a discrete Fourier projection.

\subsubsection{Bubble coefficients}

The construction of the scalar bubble coefficients is analogous with the triangle case considered above. We construct the integrand by subtracting the relevant combination of triangle and box coefficients from the double cut.

$$
\begin{aligned}
\bar{C}_{2 ; i \mid j}= & \sum_{h_{1}, h_{2}} A^{(0)}\left(-\ell_{1}^{-h_{1}}, K_{i, j-1}, \ell_{2}^{h_{2}}\right) A^{(0)}\left(-\ell_{2}^{-h_{2}}, K_{j, i-1}, \ell_{1}^{h_{1}}\right) \\
& -\sum_{k} \frac{\bar{C}_{3 ; i|j| k}\left(\ell_{1}\right)}{\left(\ell_{1}-K_{k}\right)^{2}}-\frac{1}{2} \sum_{k, l} \frac{\bar{C}_{4 ; i|j| k \mid l}\left(\ell_{1}\right)}{\left(\ell_{1}-K_{k}\right)^{2}\left(\ell_{1}-K_{l}\right)^{2}} .
\end{aligned}
$$

There are now three vectors spanning the trivial space so the integrand can be written in terms of nine independent coefficients [20],

$$
\begin{aligned}
\bar{C}_{2 ; i \mid j}= & C_{2 ; i \mid j}^{(0)}+C_{2 ; i \mid j}^{(1)} \alpha_{1}+C_{2 ; i \mid j}^{(2)} \alpha_{2}+C_{2 ; i \mid j}^{(3)} \alpha_{3}+C_{2 ; i \mid j}^{(4)}\left(\alpha_{1}^{2}-\alpha_{3}^{2}\right) \\
& +C_{2 ; i \mid j}^{(5)}\left(\alpha_{2}^{2}-\alpha_{3}^{2}\right)+C_{2 ; i \mid j}^{(6)} \alpha_{1} \alpha_{2}+C_{2 ; i \mid j}^{(7)} \alpha_{1} \alpha_{3}+C_{2 ; i \mid j}^{(8)} \alpha_{2} \alpha_{3},
\end{aligned}
$$


where $\alpha_{k}=n_{k} \cdot \ell_{1}, k=1,3$. For the massless amplitudes considered here we do not need to proceed to reduce further and extract the tadpole coefficients. The computation of $C_{2 ; i \mid j}=C_{2 ; i \mid j}^{(0)}$ completes the calculation of the cut-constructible terms. Again we use the discrete Fourier projection to efficiently compute the coefficients.

\subsection{Rational Contributions}

Using a super-symmetric decomposition of the gluonic loop [6] one can show that the rational terms for our one-loop amplitude are the same as those coming from contributions with a scalar loop. The information coming from the $4-2 \varepsilon$-dimensional cuts can be encapsulated by adding a mass parameter to the four-dimensional loop momenta,

$$
\ell_{[4-2 \varepsilon]}=\ell_{[4]}+\ell_{[-2 \varepsilon]}
$$

where $\ell_{[-2 \varepsilon]}^{2}=-\mu^{2}$. We then proceed to extract the coefficients of Eq. (5) from cuts with a massive scalar running inside the loop $[15,16,19,47,53]$. We note that the supersymmetric decomposition relates the rational part of the gluon amplitude to that of a complex scalar. Therefore, in order to match with Eq. (5), the products of trees are all multiplied by a factor of two.

\subsubsection{The pentagon coefficients}

An additional complication in the numerical computation of the $D$-dimensional pieces is the presence of a non-zero pentagon coefficient. Although such a contribution will vanish explicitly in an analytical calculation here we are forced to include them to ensure a numerically stable result. There is no trivial space for this contribution and the result appears solely as a subtraction term for the box coefficient. The coefficient is then simply [17],

$$
\begin{aligned}
& R_{5 ; i|j| k \mid l}=2 A_{S}^{(0)}\left(-\ell_{1}, K_{i, j-1}, \ell_{2}\right) A_{S}^{(0)}\left(-\ell_{2}, K_{j, k-1}, \ell_{3}\right) \\
& \times A_{S}^{(0)}\left(-\ell_{3}, K_{k, l-1}, \ell_{4}\right) A_{S}^{(0)}\left(-\ell_{4}, K_{l, m-1}, \ell_{5}\right) A_{S}^{(0)}\left(-\ell_{5}, K_{m, i-1}, \ell_{1}\right) .
\end{aligned}
$$

The five on-shell constraints can be satisfied by fixing the four coefficients of the loop momentum using,

$$
\left\{2 \ell_{1} \cdot K_{i, j-1}=K_{i, j-1}^{2}, 2 \ell_{1} \cdot K_{i, k-1}=K_{i, k-1}^{2}, 2 \ell_{1} \cdot K_{i, l-1}=K_{i, l-1}^{2}, 2 \ell_{1} \cdot K_{i, m-1}=K_{i, m-1}^{2}\right\},
$$

and additionally setting the $D$-dimensional mass by $\mu^{2}=\ell_{1}^{2}$. The implementation in NGluon chooses to implement a solution to these on-shell constraints using two-dimensional Weylspinors along the lines of those used in Refs. [8,9,19] since we found it more efficient and numerically stable than the van-Neerven basis solution used in Ref. [17]. This allows us to avoid the computation of any $4 \times 4$ determinants. 


\subsubsection{The box coefficients}

Since we compute the $D$-dimensional coefficient $C_{4}^{[4]}$ from four-dimensional massive cuts, the first part of the calculation proceeds exactly as in the cut-constructible case. The quadruple cut together with the pentagon subtraction is a polynomial in $\mu^{2}$ from which we can extract the coefficients using a discrete Fourier projection.

$$
\begin{aligned}
\bar{R}_{4 ; i|j| k \mid l}= & 2 A_{S}^{(0)}\left(-\ell_{1}, K_{i, j-1}, \ell_{2}\right) A_{S}^{(0)}\left(-\ell_{2}, K_{j, k-1}, \ell_{3}\right) \\
& \times A_{S}^{(0)}\left(-\ell_{3}, K_{k, l-1}, \ell_{4}\right) A_{S}^{(0)}\left(-\ell_{4}, K_{l, i-1}, \ell_{1}\right) \\
& -\sum_{m} \frac{C_{5 ; i|j| k|l| m}}{\left(\ell_{1}-K_{m}\right)^{2}} .
\end{aligned}
$$

The polynomial form of the integrand is,

$$
\begin{aligned}
& \bar{R}_{4 ; i|j| k \mid l}\left(\ell_{1}\left(\mu^{2}\right)\right)=R_{4 ; i|j| k \mid l}^{(0)}+n_{1} \cdot \ell_{1} R_{4 ; i|j| k \mid l}^{(1)} \\
& \quad+\mu^{2}\left(R_{4 ; i|j| k \mid l}^{(2)}+n_{1} \cdot \ell_{1} R_{4 ; i|j| k \mid l}^{(3)}\right)+\mu^{4}\left(R_{4 ; i|j| k \mid l}^{(4)}+n_{1} \cdot \ell_{1} R_{4 ; i|j| k \mid l}^{(5)}\right),
\end{aligned}
$$

where $C_{4 ; i|j| k \mid l}^{[4]}=R_{4 ; i|j| k \mid l}^{(4)}$. Performing the four-dimensional extraction three times is then sufficient to extract all the coefficients. We choose the mass integration to lie on a circle of radius $\mu_{0}$,

$$
\mu_{m}^{2}=\mu_{0}^{2} \exp \left(\frac{2 \pi i m}{3}\right)
$$

so the explicit solution becomes

$$
\begin{aligned}
& R_{4 ; i|j| k \mid l}^{(2 p)}=\sum_{m=0}^{5} \frac{1}{2 \mu_{m}^{2 p}}\left(\bar{R}_{4 ; i|j| k \mid l}\left(\ell_{1}^{+}\left(\mu_{m}^{2}\right)\right)+\bar{R}_{4 ; i|j| k \mid l}\left(\ell_{1}^{-}\left(\mu_{m}^{2}\right)\right)\right) \\
& R_{4 ; i|j| k \mid l}^{(2 p+1)}=\sum_{m=0}^{5} \frac{1}{2 \mu_{m}^{2 p} \sqrt{\Delta_{4}}}\left(\bar{R}_{4 ; i|j| k \mid l}\left(\ell_{1}^{+}\left(\mu_{m}^{2}\right)\right)-\bar{R}_{4 ; i|j| k \mid l}\left(\ell_{1}^{-}\left(\mu_{m}^{2}\right)\right),\right)
\end{aligned}
$$

with $p=0,1,2$. It is important to choose the value of the radius of integration, $\mu_{0}$, such that the quadruple cut and subtraction terms are of the same order of magnitude. There are various ways to do this. In NGluon the radius is scaled with respect to the largest pentagon contribution that occurs in the subtractions in order to maximise numerical stability. Since the rank of tensor integrals is constrained to be of maximum four in gauge theory, it will always be the case that $R_{4 ; i|j| k \mid l}^{(5)}=0$. This can be a useful test of the accuracy of the computation of $\bar{R}_{4 ; i|j| k \mid l}$.

\subsubsection{The triangle coefficients}

At this stage the method should be quite clear. We proceed to extract the triangle coefficients from the massive scalar loop, sampling over the mass parameter to find the coefficient contributing the the rational term. In order to have an integrand with polynomial behaviour we 
must subtract both pentagon and box contributions from the product of trees,

$$
\begin{aligned}
\bar{R}_{3 ; i|j| k}= & 2 A_{S}^{(0)}\left(-\ell_{1}, K_{i, j-1}, \ell_{2}\right) A_{S}^{(0)}\left(-\ell_{2}, K_{j, k-1}, \ell_{3}\right) A_{S}^{(0)}\left(-\ell_{3}, K_{k, i-1}, \ell_{1}\right) \\
& -\sum_{l} \frac{\bar{R}_{4 ; i|j| k \mid l}}{\left(\ell_{1}-K_{l}\right)^{2}}-\frac{1}{2} \sum_{l, m} \frac{R_{5 ; i|j| k|l| m}}{\left(\ell_{1}-K_{l}\right)^{2}\left(\ell_{1}-K_{m}\right)^{2}} .
\end{aligned}
$$

The polynomial structure of the integrand can be written:

$$
\begin{aligned}
\bar{R}_{3 ; i|j| k}\left(\ell_{1}\right) & =R_{3 ; i|j| k}^{(0)}+R_{3 ; i|j| k}^{(1)} \alpha_{1}+R_{3 ; i|j| k}^{(2)} \alpha_{2}+R_{3 ; i|j| k}^{(3)}\left(\alpha_{1}^{2}-\alpha_{2}^{2}\right) \\
& +R_{3 ; i|j| k}^{(4)} \alpha_{1} \alpha_{2}+R_{3 ; i|j| k}^{(5)} \alpha_{1}^{2} \alpha_{2}+R_{3 ; i|j| k}^{(6)} \alpha_{1} \alpha_{2}^{2} \\
& +\mu^{2}\left(R_{3 ; i|j| k}^{(7)} \alpha_{1}+R_{3 ; i|j| k}^{(8)} \alpha_{2}+R_{3 ; i|j| k}^{(9)}\right)
\end{aligned}
$$

where $\alpha_{1}, \alpha_{2}$ are identical to that of the cut-constructible triangle. The Fourier projection over the mass proceeds as in the box contributions except we are required to sample over more points due to the larger number of independent coefficients.

\subsubsection{The bubble coefficients}

No new features appear in the extraction of this final term in the amplitude so we simply write down the formulae for the integrand and it's polynomial structure. We point readers towards Refs. $[8,17,18,20,21]$ for further details,

$$
\begin{aligned}
\bar{R}_{2 ; i \mid j}= & 2 A_{S}^{(0)}\left(-\ell_{1}, K_{i, j-1}, \ell_{2}\right) A_{S}^{(0)}\left(-\ell_{2}, K_{j, i-1}, \ell_{1}\right)-\sum_{k} \frac{\bar{R}_{3 ; i|j| k}}{\left(\ell_{1}-K_{k}\right)^{2}} \\
& -\frac{1}{2} \sum_{k, l} \frac{\bar{R}_{4 ; i|j| k \mid l}}{\left(\ell_{1}-K_{k}\right)^{2}\left(\ell_{1}-K_{l}\right)^{2}}-\frac{1}{6} \sum_{k, l, m} \frac{R_{5 ; i|j| k|l| m}}{\left(\ell_{1}-K_{k}\right)^{2}\left(\ell_{1}-K_{l}\right)^{2}\left(\ell_{1}-K_{m}\right)^{2}},
\end{aligned}
$$

and

$$
\begin{aligned}
\bar{R}_{2 ; i \mid j}= & R_{2 ; i \mid j}^{(0)}+R_{2 ; i \mid j}^{(1)} \alpha_{1}+R_{2 ; i \mid j}^{(2)} \alpha_{2}+R_{2 ; i \mid j}^{(3)} \alpha_{3}+R_{2 ; i \mid j}^{(4)}\left(\alpha_{1}^{2}-\alpha_{3}^{2}\right) \\
& +R_{2 ; i \mid j}^{(5)}\left(\alpha_{2}^{2}-\alpha_{3}^{2}\right)+R_{2 ; i \mid j}^{(6)} \alpha_{1} \alpha_{2}+R_{2 ; i \mid j}^{(7)} \alpha_{1} \alpha_{3}+R_{2 ; i \mid j}^{(8)} \alpha_{2} \alpha_{3}+\mu^{2} R_{2 ; i \mid j}^{(9)}
\end{aligned}
$$

$\alpha_{i}$ are the same as those in Eq. (18) and the coefficient of eq. Eq. (5) is $C_{2 ; i \mid j}^{[2]}=R_{2 ; i \mid j}^{(9)}$.

\section{Installation}

NGluon uses the GNU compiler suite and is available as a tarball, NGluon-1.1.tar.gz, from http: //www. physik.hu-berlin.de/pep/tools.

If NGluon is used without QCDLOop and without the qd extension the $\mathrm{g}++$ compiler is sufficient to compile and install the package. To do this first unpack the archive using: 
You can then move to the directory NGluon-1.1 and type make. This will build the NGluon library (for static linking): libNGluon. a. The object files and library are placed into the directory NGluon-1.1 / obj. In addition an example application NGluon-demo will be created. We note that most of the files found in NGluon-1.1 belong to the sample application. In particular these files contain additional code to generate phase space points and analytic results. For the details we refer to Tab. 1 where a short description of the files is given. The upper block of the table describes the files necessary to build the library. Files in the lower block are needed only for the example applications. We will not describe files in the lower block in detail since they are only provided for illustrative purpose and are not part of the NGluon package itself.

Without a library for the scalar one-loop integrals NGluon calculates the coefficients of the scalar integrals as well as the rational part. However since all the scalar integrals are set to one the full result for the full amplitude is meaningless. As a consequence most of the tests which can be found in NGluon-demo. cpp will not work. To obtain the full functionality NGluon should be combined with a library for the evaluation of the scalar one-loop integrals. NGluon is prepared for use with FF [43] and QCDLoop [42] for the evaluation of the scalar integrals. The package has been tested with QCDLoop-1.9 which can be downloaded at the following address:

http: //qcdloop. fnal.gov/

Note that per default QCDLoop uses g77. Since g77 is no longer supported we recommend to switch to gfortran. If g77 shall be used the user needs to adopt the makefile in NGluon-1.1 . In particular the linker options have to be adjusted to enable the linking of code compiled with the fortran compiler together with the main program compiled with g++. To use QCDLoop the user should first install the QCDLOop library. For the details how to do this we refer to the QCDLoop documentation.

To link QCDLoop with NGluon it is sufficient to edit the configuration of NGluon which is controlled via the file: Makefile.inc. To include QCDLoop the variable ENABLE_QL is changed to ENABLE_QL=yes. In addition the path to the QCDLoop-1.9 installation (the QCDLoop-1.9 directory) needs to be be configured through the variable QLDIR in Makefile. inc. Typing,

make clean

make

will then compile a version of NGluon including the scalar one-loop integrals from the QCDLoop library.

NGluon is also prepared to work with extended floating point arithmetic as provided for example by the qd library [54]. The library can be obtained at the following address:

http://crd.lbl.gov/ dhbailey/mpdist/

NGluon has been tested with version $q d-2.3 .11$.

Once the qd library has been installed one may easily compile quadruple and octuple precision versions of NGluon via Makefile.inc. Simply change ENABLE_DD=yes ("double-double") 
NGluon library

\begin{tabular}{l|l}
\hline File name & Functionality \\
\hline NGluon.h & Header file for NGluon \\
NGluon.cpp & Source file with the implementation of the unitarity method \\
Current.h & Header file for Berends-Giele related functions \\
Current.cpp & Implementation of the Berends-Giele recursion \\
Coefficients.h & Definition of storage used internally \\
LoopIntegrals.cpp & Interface to QCDLoop \\
LoopIntegrals.h & Interface to QCDLoop \\
mytypes.h & Header file to switch to extended precision using qd \\
\hline
\end{tabular}

Sample application

\begin{tabular}{l|l}
\hline File name & Functionality \\
\hline analytic.h & Header file for analytic formulae \\
analytic.cpp & Implementation of some analytic formulae \\
NGluon-demo.cpp & Example application \\
GKM.cpp & Results from Ref. [17] \\
GKM.h & Results from Ref. [17] \\
GZ.cpp & Results from Ref. [22] \\
GZ.h & Results from Ref. [22] \\
FourMomentum.h & Four momentum class \\
histogram.h & Simple histogram functionality \\
phasespace.cpp & Phase space generation \\
phasespace.h & Phase space generation \\
\hline
\end{tabular}

Make files

\begin{tabular}{l|l}
\hline File name & Functionality \\
\hline Makefile & Makefile to built the libraries and compile the example applications. \\
Makefile.all & Makefile specific instances of the library and create driectory structure \\
Makefile.inc & Configuration file for the Makefile
\end{tabular}

Table 1: Files included in the NGluon package. In the upper part files belonging to the NGluon library itself are listed. In the lower part files which are only used by the example application NGluon-demo are shown. 
or ENABLE_QD=yes ("quad-double") in the configuration file Makefile.inc. The path to the library and the location of the header files needs to be configured via QDLIB and QDINCLUDE. Again,

make clean

make

will then compile the relevant versions of the NGluon library placing the library and object files into the directories NGluon-1.1 / obj, NGluon-1.1 / obj-dd and NGluon-1.1 / obj-qd. Up to three versions of the test program are also created in NGluon-1.1: NGluon-demo, NGluon-demo-dd and NGluon-demo-qd. Since the object files are put into different directories, the different versions do not interfere with each other and can be used in parallel. Note that the floating point arithmetic used in QCDLoop is not changed. In particular, if numerical instabilities arise in the evaluation of the scalar integrals they would not be cured by switching to extended precision.

If specific compiler options are required these options must be added also in Makefile.inc via the CFLAGS variable. Non-standard locations for other libraries may be added to LIBS, LFLAGS and IFLAGS.

One can switch the compiler via the standard makefile variable CXX. The degree of optimisation can be changed via OPT though we recommend -02 .

\section{Description}

We decided to encapsulate the entire implementation in a class called NGluon. The main purpose of this approach is to hide most of the internal data required to store partial results from the user. To instantiate an object of the NGluon class the following constructor is used (the only one available):

NGluon loop_amp (ngluon, moms, helicities) ;

where ngluon is an integer denoting the number of gluons, and moms specifies a pointer to an array containing the momentum configuration with the momenta counted outgoing. The corresponding $\mathrm{C}++$ definition would be:

DOUBLE moms [ngluon] [4] .

Note that we use everywhere the preprocessor macro DOUBLE instead of the built-in data type double. Using the qd library [54] this allows us to create a version of the program using extended floating point arithmetic by simply recompiling the program. The header file mytypes.h takes care to set the macro DOUBLE to the required value that is either dd_real 
or qd_real when compiling with extended precision or double when built-in double precision shall be used. Instead of the preprocessor variable to control the data type, we could have used $\mathrm{C}++$ templates. However, code generation via the compiler is much harder to control in this case. In addition this approach often leads to longer executables which may affect the performance in a negative way. The last argument in the constructor specifies an integer array where the helicities $( \pm 1)$ for the gluons are stored. The corresponding $\mathrm{C} / \mathrm{C}++$ definition reads:

int helicities[ngluon].

Note that these arrays are not copied by the NGluon class, only the address of the arrays is stored in the NGluon object. After updating the momentum configuration or the helicity configuration NGluon will thus automatically use the updated quantities in the next call. We note also that it is not foreseen to change the number of gluons after the NGluon object has been constructed. To study amplitudes with differing numbers of gluons a new instance must be constructed for each case. Since all local data is stored inside the class, these instances do not interfere with each other. In principle the class itself should also be thread-safe.

Below we give a list of all public methods together with a short description.

static void setVerbosity (VERBOSITY output_);

This function controls the verbosity of the NGluon class. Using NGluon: : QUIET as argument turns all debugging information off while NGluon: : FULL switches to maximal verbosity.

void setMuR (DOUBLE muR_);

Used to set the renormalisation scale used in the scalar one-loop integrals. Per default the renormalisation scale is set to 1 .

void setScaleTest (bool scaleTest_);

If the argument is true the function switches the scale test on. For a detailed description see below. We note that if the scale test is switched on the runtime doubles, however a reliable estimate for the accuracy is provided for the final result. By default the scale test is switched off.

std: : complex<DOUBLE> evalAmp ();

Calling this function will evaluate the n-gluon amplitude for the momentum and helicity configuration provided in the constructor. The return value is the finite part of the amplitude.

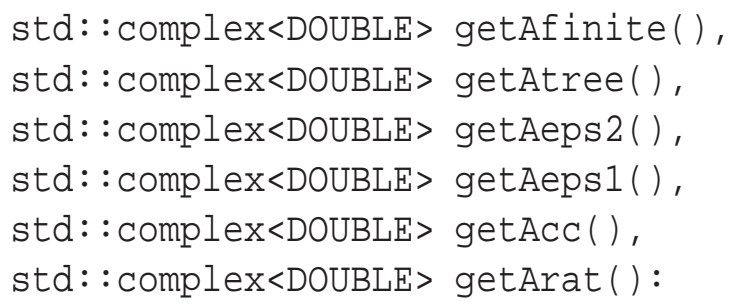

These functions give access to the finite part of the one-loop amplitude as well as to individual contributions like the value of the corresponding tree amplitude, the $1 / \varepsilon^{2}$ - and $1 / \varepsilon$-poles as well as the cut-constructible (cc) and the rational part (rat). 
std: : complex<DOUBLE> getAbsError (),

std: : complex<DOUBLE> getAbsErrorEps1 (),

std: : complex<DOUBLE> getAbsErrorEps2 (),

std: : complex<DOUBLE> getAbsErrorRR () :

these functions provide an estimate for the absolute uncertainty of individual contributions. For the details how these estimates are obtained see the discussion at the end of this section.

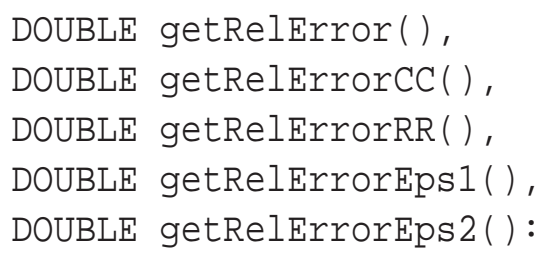

Similar to the functions described above. However instead of an estimate for the absolute uncertainty the relative uncertainty is returned.

DOUBLE IRpoles (const int eps);

This function returns the IR poles obtained from the analytic formulae (see Eq. (6)) for the given momentum and helicity configuration. The return value is the pre-factor multiplying the corresponding born amplitude without the pole itself. For eps $=-2$ the $1 / \varepsilon^{2}$-pole is returned. For eps $=-1$ the $1 / \varepsilon$-pole is returned. The result is used as a cross check of the results obtained by NGluon from the direct numerical evaluation.

static void sethel (int ngluon, int htype, int helicity[]) ;

The function creates specific helicity configurations for ngluon gluons. The configuration is stored in the array specified as third argument. More specifically the configurations are:

\begin{tabular}{|l|l|}
\hline htype & configuration \\
\hline 0 & $(+)^{n}$ \\
\hline 1 & $-(+)^{n-1}$ \\
\hline 2 & $--(+)^{n-2}$ \\
\hline 3 & $(-+)^{n / 2}$ \\
\hline 4 & $(+-)^{n / 2}$ \\
\hline
\end{tabular}

static void sethel (int ngluon, std: string hstr, int helicity[]);

The function sets the helicity configuration for ngluon gluons specified through a string in the form $"+-++-. . . "$.

static std::string helicity2string(const int ngluon, int hel[]);

The function converts a helicity configuration for ngluon gluons specified through the integer array int hel [] into a string.

A crucial point in the numerical evaluation of one-loop amplitudes is the control of numerical uncertainties and instabilities. To assure the correctness of the calculated scattering amplitudes we need checks to test the reliability of the results. Where analytic results are known, we may compare with them and we discuss such comparisons in the next section. However in most cases analytic results are not available. In such cases important information can be obtained 
from testing general properties of the amplitudes. Such a test is for example provided by the evaluation of the IR poles of the amplitude. The IR poles are analytically known due to the universal structure of IR phenomena in QCD. We can thus compare what we obtain from the numerical evaluation with what is predicted by QCD. This check tests the coefficients of the IR divergent triangle and box integrals. More precisely a specific linear combination of these coefficients is checked. Similar the UV structure which is also known analytically can be used as a test. This check provides information on the coefficients of the two-point integrals. Testing the remaining parts of the cut-constructible contribution to the full amplitude as well as the test of the rational part is more involved. Useful information may be obtained from the evaluation of the Fourier projection. It is possible to calculate further terms in the Fourier projection which are predicted to be zero. One can then check to what extend the numerical results are compatible with zero. This gives important information on the accuracy of the Fourier projection. An important property of this check is that the additional computing effort is moderate. However, the interpretation of the result of this check in terms of errors on specific coefficients might be non-trivial in particular when large cancellations between individual coefficients take place. Since a solid error estimate is crucial, we developed a simple but very effective method to get a reliable estimate. It is based on the simple observation that we can rescale the momenta and recalculate the amplitude. Owing to the rescaling we call this test scale test. From a physical point of view the test corresponds to use two different units in specifying the momenta, i.e. $\mathrm{MeV}$ and $\mathrm{GeV}$ for example. Since we know how the amplitudes scale when we rescale the momenta it is possible to compare the two results with each other. Naively, one could expect to get precisely the same result. This could be true even in the presence of rounding errors if the scaling would only affect the exponent of the floating point representation. However, rescaling with a factor which cannot be absorbed into a shift of the exponent in the binary representation of the floating point number will lead to a different mantissa. The floating point arithmetic thus becomes different. That is digits in the final result which are affected by rounding errors or numerical instabilities will change. That gives us a very simple method to check the reliability of individual contributions to the amplitude even for contributions where no analytic results are available. We should mention that this luxury comes at the price of a doubled runtime since every phase space point is calculated twice. For dedicated comparisons between different codes or with analytic formula we feel however that the effort is well spent. In practical applications one would use the scale test only for phase space points where we have indications - based on the checks described before - that the result might be unreliable. If the scale test leads to the result that the point is reliable this procedure is less computing extensive than switching to extended accuracy which would be done only if the scale test leads to the conclusion that the accuracy does not meet the requirements. As described above the test can be switched on and off using the function set ScaleTest. We will illustrate the scale test in the next section. 


\section{Usage and examples}

Before discussing the performance of the implementation, let us first present a simple example to illustrate how the package is used. All results of this section were produced using the program NGluon-demo which is included in the package. The user can thus easily reproduce the numerical results presented in this article. The file NGluon-demo. cpp may also serve in providing further examples how to use NGluon. The following example which we will discuss in detail is taken from the routine GZcheck in NGluon-demo.cpp. The routine compares results obtained with NGluon with results given in Ref. [22]:

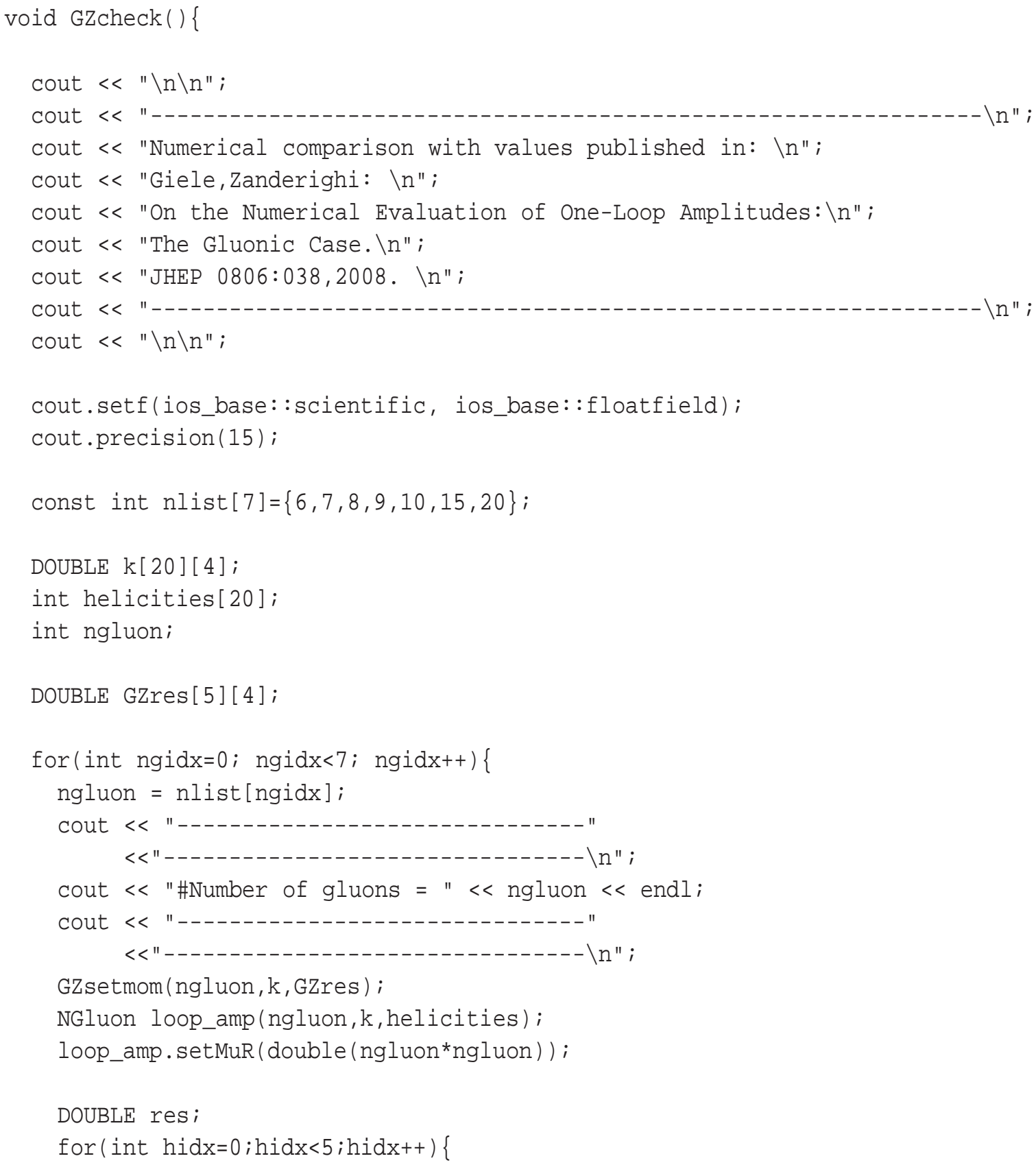




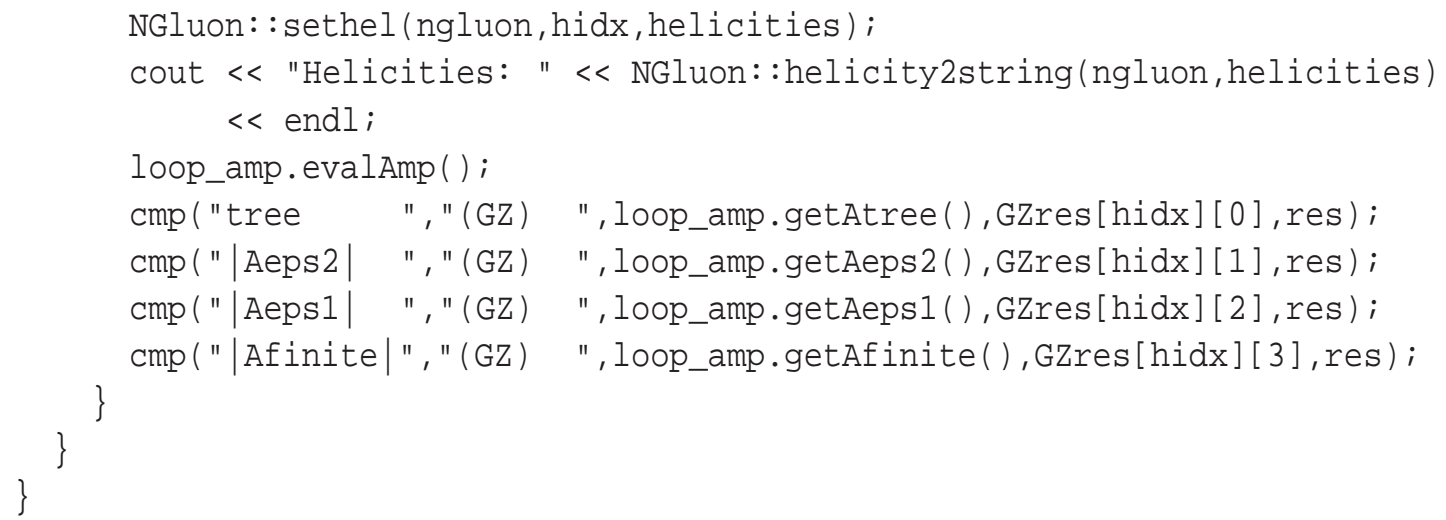

The arrays DOUBLE $\mathrm{k}[20][4]$ and int helicities [20] are used to store the momentum and helicity configuration as described before. We loop over the examples of $6,7,8,9,10,15$, and 20 gluons as presented in Ref. [22]. Inside the loop we load first the momentum configuration as specified in Ref. [22] by calling GZsetmom (ngluon, k, GZres). The function GZsetmom (ngluon, k, GZres) loads also the results as given in Ref. [22] (Tab. 1 - Tab. 7 in Ref. [22]). The next step is then to create an object of the NGluon class. The renormalisation scale is set to the value used in Ref. [22] and the scale test is switched off. It follows a loop over the different helicity configurations used in Ref. [22]. The configurations are set using NGluon: : sethel (ngluon, hidx, helicities). By calling loop_amp.evalAmp () the matrix element for the specific configuration is evaluated. In the next lines the individual contributions to the amplitude are retrieved from the NGluon class and compared with the results as shown in Ref. [22]. Compiling the file NGluon-demo.cpp this test can be run through the command line option --GZcheck. The result from the comparison will be printed on the screen. An example run is shown in the appendix A.1. For small number of gluons we find good agreement with Ref. [22]. For the examples with a larger number of gluons the agreement is getting worse. The examples for high multiplicities were calculated in extended precision in Ref. [22]. We also tried to switch to extended precision, however we do not observe a significant improvement. We believe that this is due to the fact that the momentum configuration is only given with double accuracy. Since momentum conservation and on-shellness are satisfied only to 15 digits switching to extended accuracy does not give significant improvement in this case. Using the option --GKMcheck the program will compare with results published in Ref. [17]. The sample output is shown in appendix A.2. In case of the 5 gluon amplitude we observed a discrepancy for the helicity configurations --+++ and -+-++ . We believe that this is due to a mismatch in the helicity labeling since we get agreement when we flip the helicities to ++--- and +-+-- . 


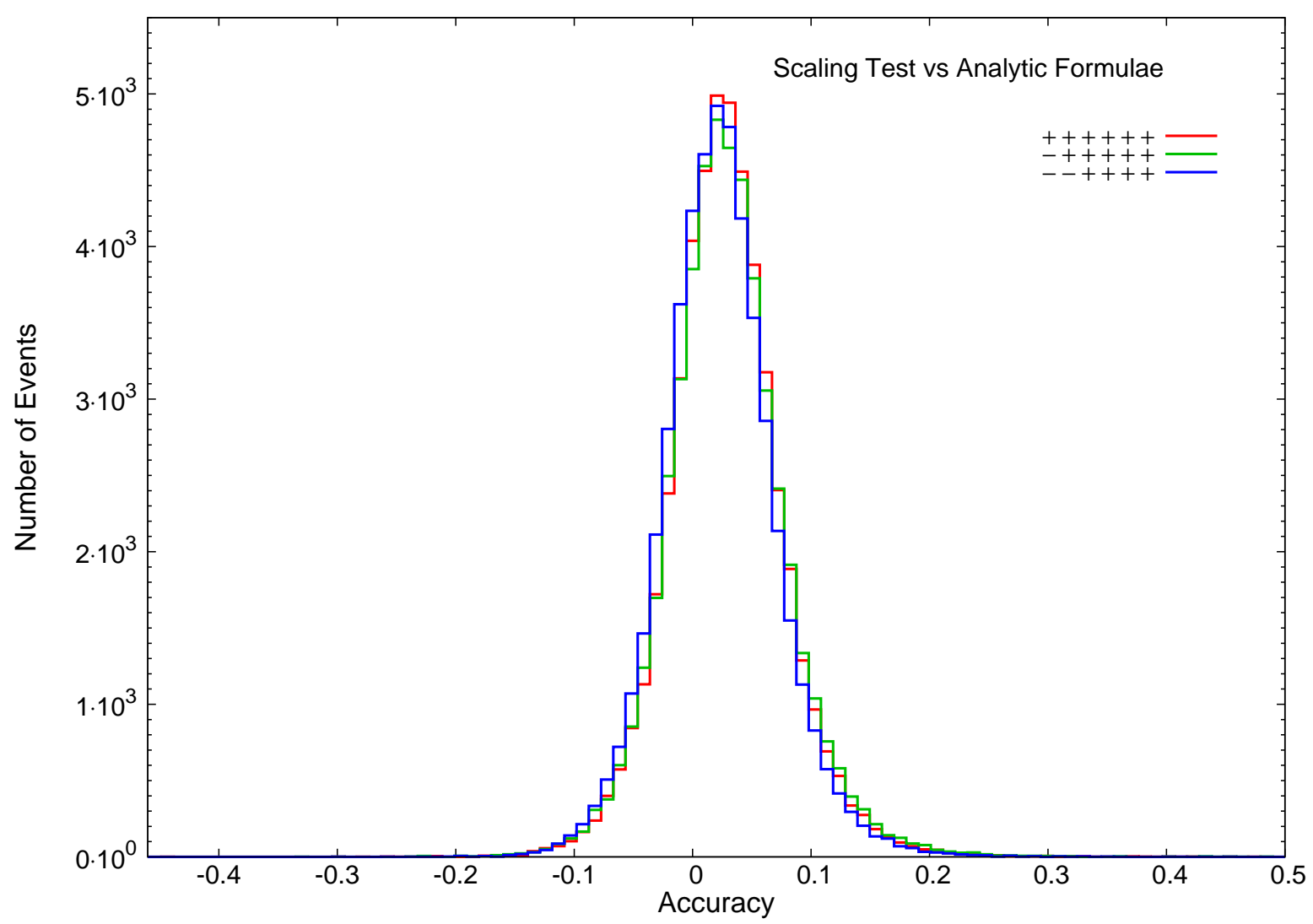

Figure 2: Test of the accuracy estimated from the scale test. 


\subsection{Accuracy}

\subsubsection{The scale test}

Although NGluon can be compiled to work with extended precision all checks in this section are obtained in double precision. This gives a direct measure for the numerical stability of the program. In the practical application one may resort to extended floating point precision, to recalculate phase space points which could not be calculated in double precision. Since some of the checks make use of the scale test described in the previous section we first assess the question how reliable this test is. To do so we study a 6-gluon amplitude, where analytic results for different helicity configurations are available $[10,12,19,55,56]$. For the helicity configurations $(+)^{6},-(+)^{5}$ and $--(+)^{4} 50000$ phase space points passing the same cuts as used in Ref. [17] were generated. The kinematic cut is applied to restrict the phase space points to the "physical region" avoiding soft and collinear configurations which may introduce further numerical instabilities. Note that in a real application the IR safe jet algorithm would provide this cut. For the 50000 phase space points the matrix elements were calculated using NGluon with the scale test switched on. In addition also the analytic formulae are used to calculate the matrix element. We estimate the absolute uncertainty $\delta_{s}$ from the scale test by taking the difference of the two results (after rescaling). The absolute uncertainty estimated from the comparison with the analytic result is defined as the difference of the numerical result and the result obtained from the evaluation of the analytic formula. The absolute uncertainties are converted to relative ones and the absolute value is taken. The logarithm of the relative uncertainty provides an easy measure for the accuracy:

$$
d_{s}=\log \left(2 \frac{\delta_{s}}{A_{1}+A_{2}}\right)=\log \left(2 \frac{A_{1}-A_{2}}{A_{1}+A_{2}}\right)
$$

and

$$
d_{a}=\log \left(2 \frac{\delta_{a}}{A_{1}+A_{a}}\right)=\log \left(2 \frac{A_{1}-A_{a}}{A_{1}+A_{a}}\right)
$$

where $A_{i}$ are the two numerical results and $A_{a}$ the one from the evaluation of the analytic formula. In the ideal case the two uncertainties would be $100 \%$ correlated. In Fig. 2 we show the distribution of

$$
\frac{d_{s}}{d_{a}}-1
$$

As one can see most of the events are located close to zero. The scale test gives thus a reliable estimate for the uncertainty. It is clear that the two methods to assess the uncertainty will not return precisely the same result. However, from the small width of the distribution we conclude that the scale test can replace the analytic comparison when no analytic results are available. Inspecting Fig. 2 in detail we observe that the distribution is slightly shifted to the right. This shift could be due to the details of the floating point arithmetic combined with the fact that we assume that the results based on the analytic formulae are always correct. For events where we estimate an accuracy of ten or even more digits the evaluation of the analytic formula may also not be precise enough to compare with. Another effect might be 
that accidentally we may estimate for a specific event a higher accuracy than we actually have. It is possible that just by chance a digit which is already out of numerical control agrees. This could happen in the comparison with the analytic result as well as in the scale test. Due to the prejudice that the analytic results are always correct this could lead to a shift in one direction.

\subsection{2 n-point MHV amplitude}

To perform further checks on the correctness of the code and also the performance with respect to numerical accuracy/stability we analysed the accuracy for different phase space points for amplitudes of different complexity. More specifically, we consider the case of $n=4,5,6,8,10,12$ gluons. The number of phase space points for each case is fixed through the requirement that each test should be done in less than a couple of hours. (For 4-6 gluons we used 1000000 phase space points, for 8 gluons we used 200000, for 10 gluons 50000 and for 12 gluons 30000 phase space points were used.) We analysed the accuracy for the $1 / \varepsilon^{2}, 1 / \varepsilon$ poles as well as the accuracy for the finite part. The events were binned according to the accuracy. We take again the logarithm of the relative uncertainty as a measure for the accuracy. The result is shown in Fig. 3. We considered the MHV amplitudes since analytic results for the rational part exist. The rational part is numerically the most complicated contribution. The accuracy can thus be taken as a pessimistic point of view. In all cases - even for high multiplicities - we observe that the leading IR singularities can be determined with high accuracy. The accuracy is never worse than -7 . For the 4 gluon amplitude we find that the different contributions - pole parts and finite parts - show similar behaviour. The accuracy is sufficiently good for most of the phenomenological applications at the LHC (if not for all). However, since in that case also analytic results are available this is not of any practical use. Beyond $n=4$ the distributions show a similar behaviour for different number of gluons. The $\frac{1}{\varepsilon}$-poles follow to some extend the finite parts. With increasing number of gluons the peak of the distributions is shifted to the right. With more gluons the computation is getting more involved, and the average accuracy decreases. Due to the logarithmic scale the histograms may be misleading. We note that for the most complicated case shown in Fig. 3 - the 12 gluon amplitude - only about $3 \%$ of all events have an accuracy above -3 . In table Tab. 2 we show the fraction of events with an accuracy above -3 . In all cases the fraction shown is evaluated

\begin{tabular}{c|l}
$n$ gluons & bad points [\%] \\
\hline 4 & - \\
5 & 0.03 \\
6 & 0.06 \\
8 & 0.2 \\
10 & 0.8 \\
12 & 3.
\end{tabular}

Table 2: Fraction of events with an accuracy above -3 .

for the $--(+)^{n-2}$ configuration. Considering different configurations may change the frac- 

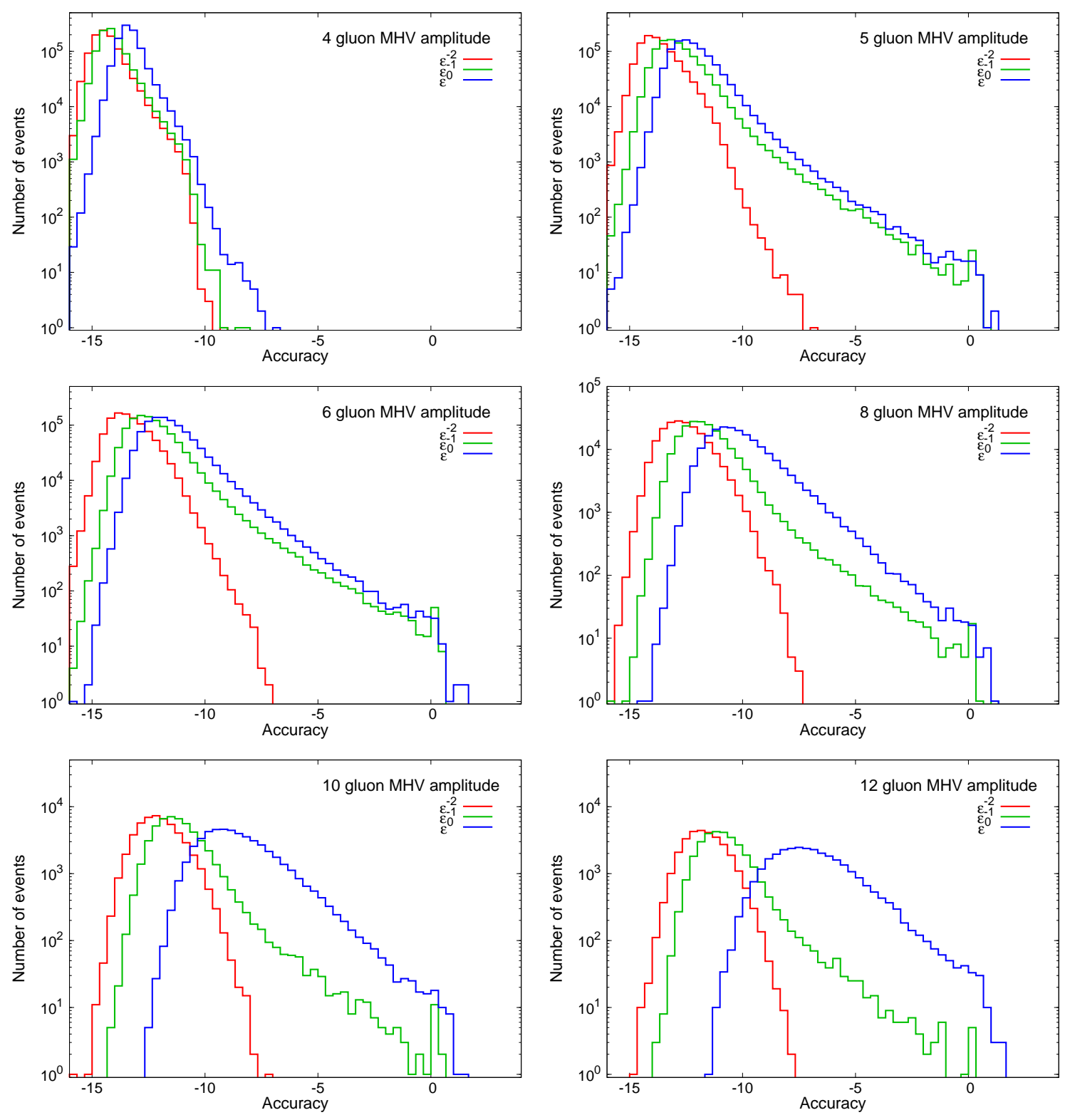

Figure 3: Accuracy for the $\frac{1}{\varepsilon^{2}}$-pole, $\frac{1}{\varepsilon}$-pole and the finite part. 
tions. We also observed that asking for an accuracy of -4 will actually double the fraction of bad points. This would mean that for the 12 gluon case, for example, about $6 \%$ of the events need to be reevaluated with higher accuracy. In Fig. 4 we show the average accuracy evaluated for a fixed number of phase space points. The accuracy is evaluated using the scale test. As we can see the accuracy is a linearly raising function of the number of gluons. Starting at $n=4$ with an average accuracy of about 12 digits, the accuracy reaches -4 for about 14 gluons. For 14 gluons we have thus on average only $3-4$ digits which are significant. To estimate whether the program can still be run mostly with built-in double precision in addition to the average accuracy the width of the distribution is important. The width is illustrated as a blue band. We observe in Fig. 4 that the width increases as a function of the number of gluons. However, the effect is only moderate in size. Assuming that for most LHC applications 4 significant digits should be sufficient we can conclude from Fig. 4 that up to 12-14 gluons the program may be used with only a small fraction of points requiring a recalculation using extended precision.

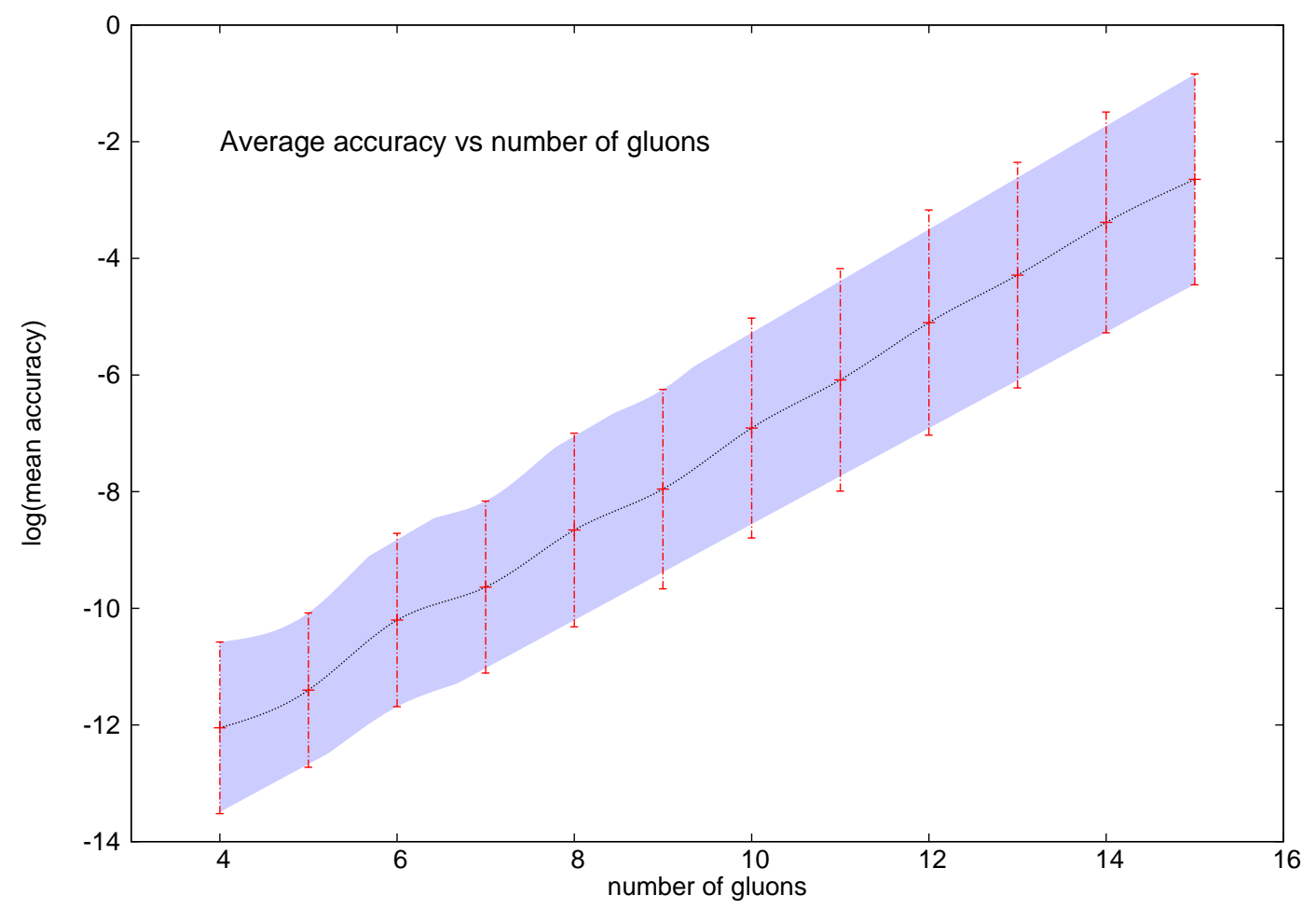

Figure 4: The average accuracy as estimated from the scale test as function of the number of gluons. The band gives a measure for the width of the distribution

One may ask the question how much the findings on the accuracy shown before depend on the specific helicity configuration. In Fig. 5 we show a similar plot as discussed before but now for the NMHV configuration. We observe that leading $1 / \varepsilon^{2}$ singularity is changed compared to what we have seen before. Since the pole part is known analytically this has no practical consequences. Inspecting the accuracy of the $\frac{1}{\varepsilon}$ pole as well as the accuracy of the finite part we observe a behaviour similar to what has been shown in Fig. 3. In Fig. 6 we show the accu- 


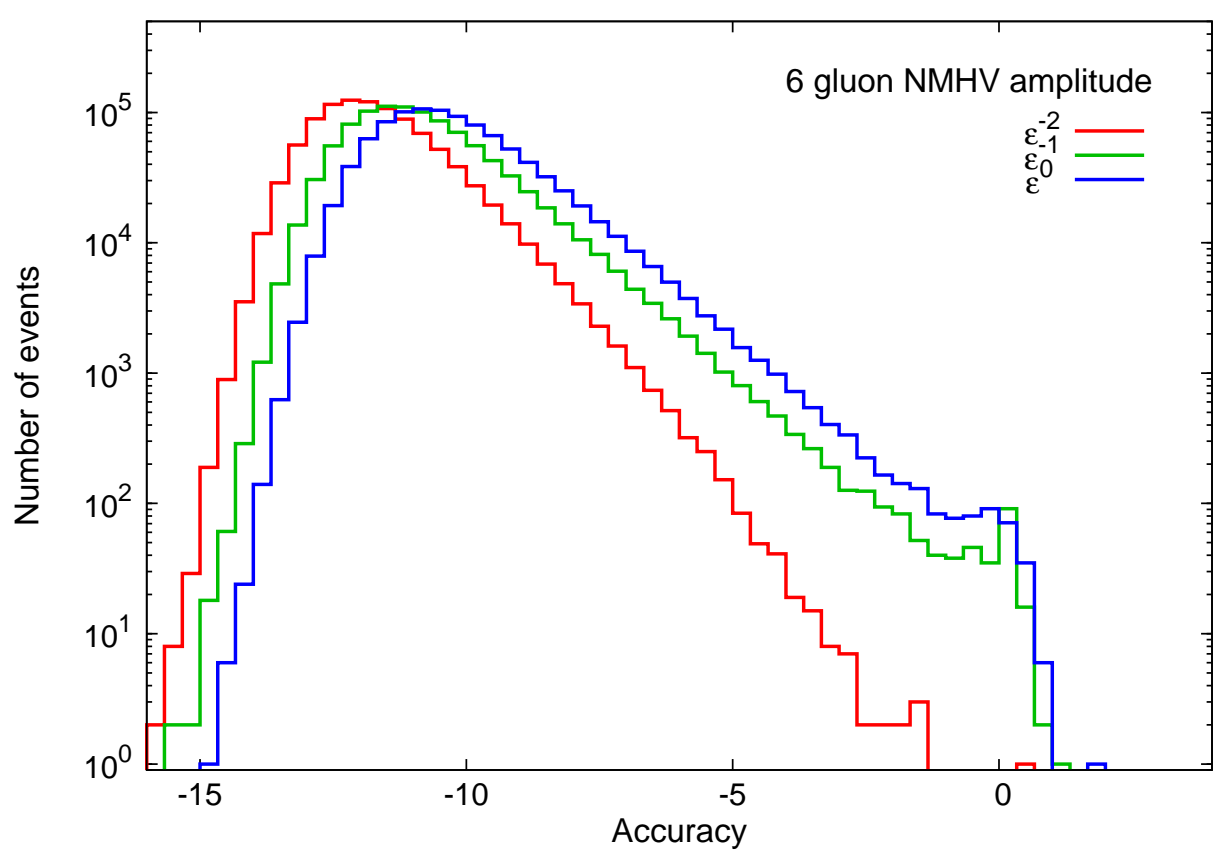

Figure 5: Accuracy for a NMHV amplitude for 6 gluons.

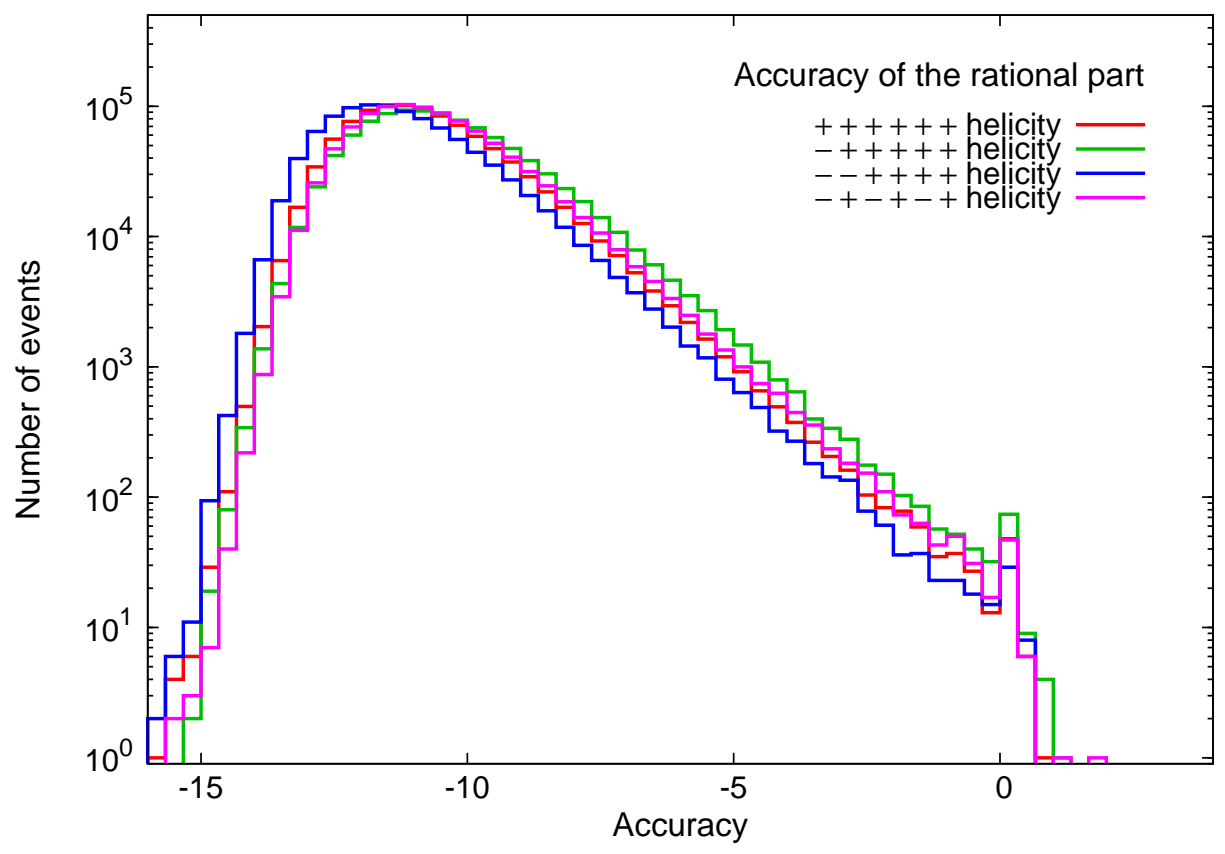

Figure 6: Accuracy of the rational part for the 6 gluon amplitude for different helicity configurations. 
racy of the rational part for different helicity configurations. Apart from minor differences at the right end of the histograms - which may be due to statistical fluctuations - we observe that the behaviour is largely independent from the chosen helicity configuration. To good approximation we thus believe that our findings are to large extend universal.

\subsection{Estimated speed}

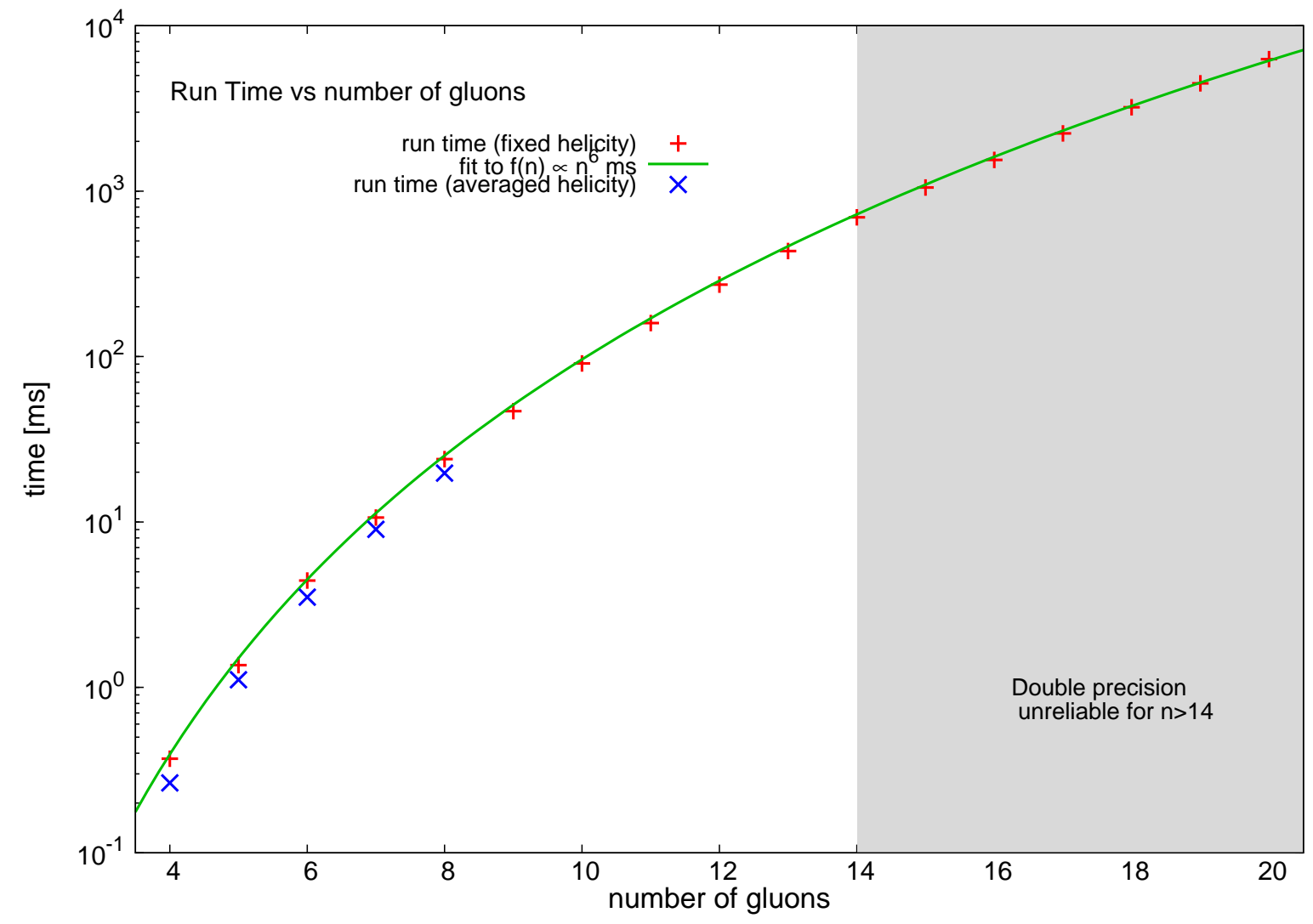

Figure 7: Runtime in milli seconds for the evaluation of one phase space point. The red crosses show the runtime for the helicity configuration which we consider the worst case. For a comparison we also show for low multiplicities an average over all possible helicity configurations (blue crosses).

In Fig. 7 we show the runtime in milli seconds required to evaluate one phase space point. The values were obtained by averaging over several evaluations to avoid large fluctuations. As hardware we used an Intel Quad Core CPU (Q9650) with a frequency of $3 \mathrm{GHz}$. As an operating system we used Scientific Linux Version 5.2. The program has been compiled with the GNU compiler suite version 4.1. The red crosses show the runtime for the evaluation of $(-+)^{n / 2}$ amplitudes. In principle, the runtime can depend on the helicity configuration since for specific cases some of the Born amplitudes entering the calculation may vanish. The 
helicity configuration shown here should correspond to the worst case. For low multiplicities we have also checked the runtime by averaging over all possible helicity configurations. We find indeed that the average runtime obtained in this way are smaller than the ones measured for the $(-+)^{n / 2}$ amplitudes. Since with increasing multiplicity the number of different helicity configurations becomes quite large, we have restricted this analysis to $n \leq 8$.

In Ref. [22] the authors showed that the computational costs behave as $n^{9}$. Due to the cache system used for the evaluation of tree amplitudes we expect to find $n^{8}$. In Fig. 7 the runtime is shown as function of the number of gluons. It turns out that for $n=20$ the asymptotic regime of large $n$ is not yet reached and we observe, in contrast to the expectation, a scaling like $n^{6}$. Extending the analysis to extreme values for $n$ we find indeed that the scaling approaches $n^{8}$. In particular we have checked that the pentagon contributions follow the $n^{8}$ behaviour as expected. However the numerical coefficient in front of the $n^{8}$ polynomial is supressed so we do not observe the $n^{8}$ behaviour for small $n$ when this contribution is combined with the remaing ones. Since running in double precision the results for $n>20$ are unreliable we have restricted ourselves to 20 gluons in Fig. 7. One should thus not take the $n^{6}$ behaviour as asymptotic scaling for large $n$. The $n^{6}$ behaviour is represented in Fig. 7 by the solid green line. Note that the region $n>14$ is shown for illustrative purpose only. In this region the numerical accuracy is so bad that a significant fraction of phase space points would require a re-evaluation using extended floating point arithmetic.

The data used in Fig. 7 can be obtained by running the program NGluon-demo with the option --runtime. We believe that further improvements are possible. In particular NGluon is based on the evaluation of tree amplitudes using the Berends-Giele recursion [49]. In the implementation of the tree amplitudes we have not used the fact that we are working in the helicity basis. This could give a further improvement. We stress that in our code no use of explicit analytic formulae for specific helicity configurations is made. This could give a further improvement.

\section{Conclusions}

We have presented a purely numerical implementation of one-loop $n$-gluon amplitudes. The implementation makes use of the generalised unitarity method developed recently by various groups. We have performed extensive checks on the numerical accuracy as well as the performance with respect to the computing time. The program has passed all these checks, is easy to use and shows a good behaviour with respect to the evaluation speed. We believe that the program can be used to obtain reliable predictions for the LHC. To the best of our knowledge the program is the only available public code which allows the numerical evaluation of arbitrary $n$-gluon one-loop amplitudes without requiring additional external input. Apart from the physical application, the package may serve as a reference implementation for future developments. In particular, it would be extremely useful to extend to full QCD processes. The extension to include fermionic loops is foreseen for the next major release. 


\section{Acknowledgments}

We would like thank Pierpaolo Mastrolia and Francesco Tramontano for illuminating discussions. We are also grateful to Valery Yundin for advice and testing of the code. This work is supported in part by the Deutsche Forschungsgemeinschaft through the Transregional Collaborative Research Centre SFB-TR9 "Computational Particle Physics"and the Research Training Group (GK1504) "Mass, Spectrum, Symmetry, Particle Physics in the Era of the Large Hadron Collider" GK1504. In addition we acknowledge support from the Helmholtz Alliance "Physics at the Terascale" contract VH-HA-101. The work of SB has been supported in part by Danish Natural Science Reseach Council grant 10-084954.

\section{A Sample output}

\section{A.1 Comparison with Giele, Zanderighi}

Running the sample application NGluon-demo with the option --GZcheck should produce output similar to what is listed below:

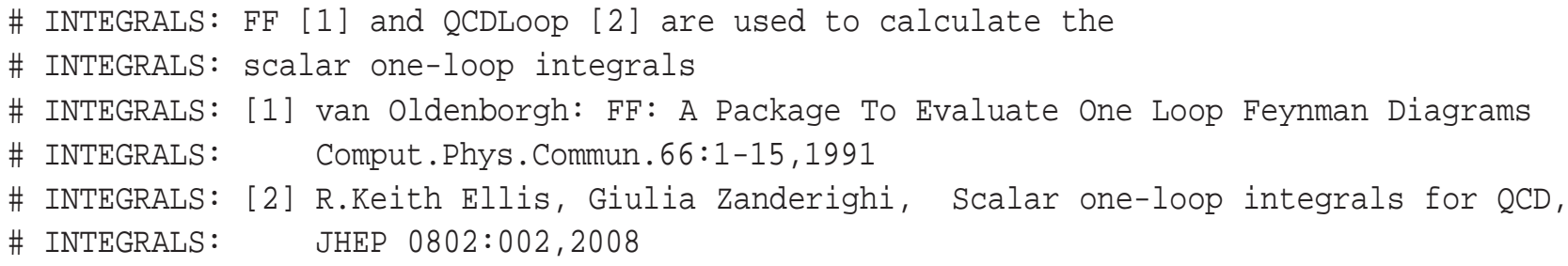

Numerical comparison with values published in:

Giele, Zanderighi:

On the Numerical Evaluation of One-Loop Amplitudes:

The Gluonic Case.

JHEP $0806: 038,2008$.

\#Number of gluons $=6$

\# ONELOOP: Renormalization scale set to $\mathrm{mu}=3.600000000000000 \mathrm{e}+01$

Helicities: ++++++

tree $\quad: 2.449772238656663 e-15$

tree (GZ) : $0.000000000000000 e+00$

|Aeps2 $\quad: 0.000000000000000 e+00$ 


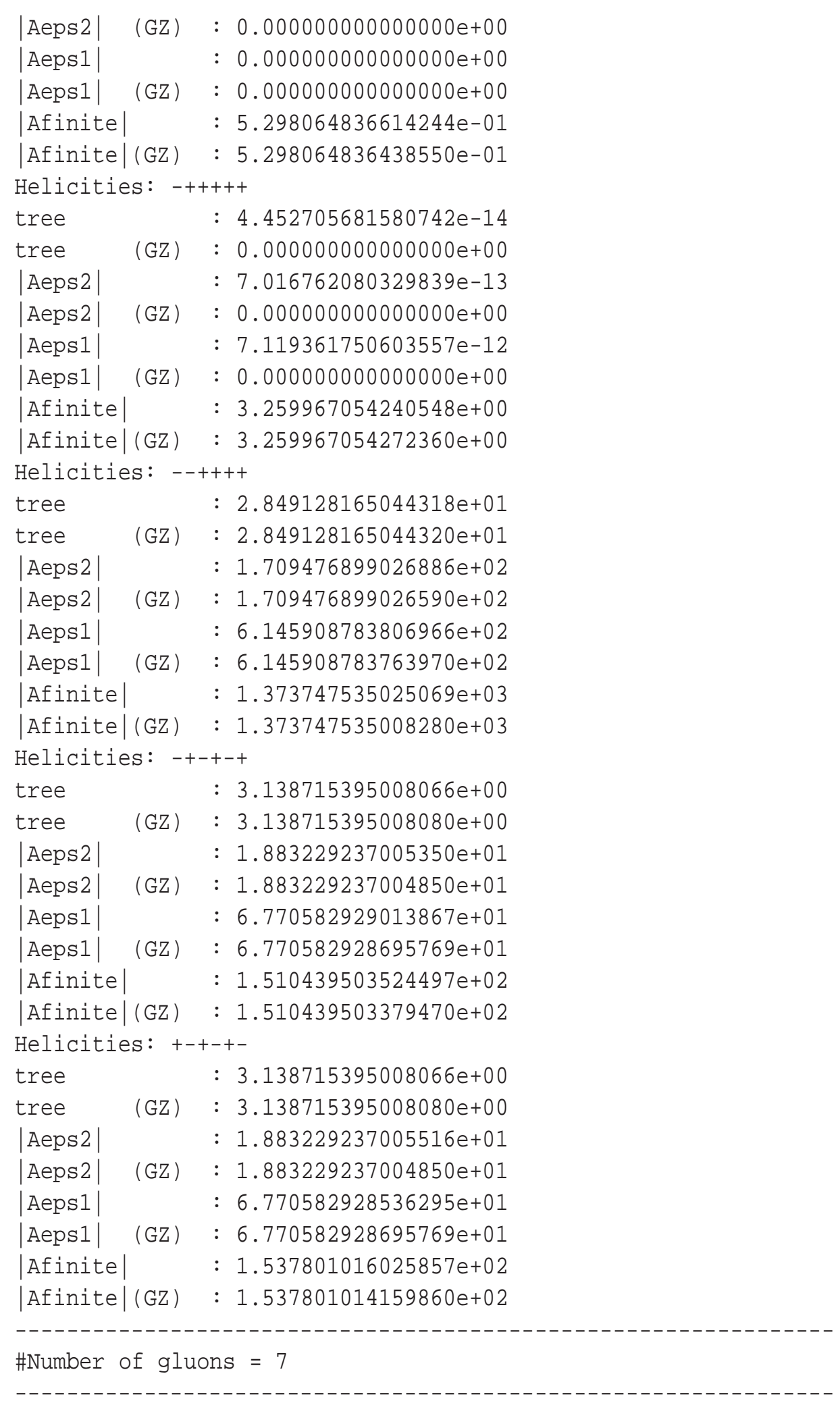

\# ONELOOP: Renormalization scale set to $\mathrm{mu}=4.900000000000000 \mathrm{e}+01$ Helicities: +++++++ 


\begin{tabular}{|c|c|c|}
\hline cee & & $: 1.164850097354693 e-15$ \\
\hline ee & (GZ) & $: 0.000000000000000 \mathrm{e}+00$ \\
\hline Aeps2 & & $: 0.000000000000000 \mathrm{e}+00$ \\
\hline Aeps2 & (GZ) & $: 0.000000000000000 \mathrm{e}+00$ \\
\hline ps1 & & $: 0.000000000000000 e+00$ \\
\hline Aeps1| & (GZ) & $: 0.000000000000000 e+00$ \\
\hline Afinite & & $: 3.101695333690422 \mathrm{e}-01$ \\
\hline finite| & (GZ) & $: 3.101695334831830 \mathrm{e}-01$ \\
\hline licitie & & \\
\hline tree & & $: 1.414070990000$ \\
\hline tree & (GZ) & $: 0.000000000000000 \mathrm{e}+00$ \\
\hline |Aeps2 | & & : 2.393016434615393e-14 \\
\hline |Aeps2 & (GZ) & $: 0.000000000000000 e+00$ \\
\hline Aeps1 1 & & $: 1.074107565329303 \mathrm{e}-13$ \\
\hline |Aeps1 $\mid$ & (GZ) & $: 0.000000000000000 \mathrm{e}+00$ \\
\hline Afinite| & & $: 1.920528150920153 \mathrm{e}-01$ \\
\hline |Afinite| & (GZ) & $: 1.92052814$ \\
\hline Helicit & & \\
\hline tree & & $: 2.10$ \\
\hline tree & (GZ) & : $2.106612834594490 \mathrm{e}+00$ \\
\hline |Aeps2 $\mid$ & & : 1.47462898421 \\
\hline |Aeps2 & (GZ) & $: 1.474628984$ \\
\hline Aeps1 1 & & $: 4.850089396$ \\
\hline Aeps1 1 & (GZ) & $: 4.850089396$ \\
\hline |Afinite| & & $: 8.731521551$ \\
\hline Afinite & (GZ) & $: 8.731521551$ \\
\hline Helicitie & $S:-+$ & -+-+- \\
\hline tree & & $: 1.10$ \\
\hline tree & (GZ) & $: 1.101865680$ \\
\hline |Aeps2 | & & $: 7.713$ \\
\hline |Aeps2 & (GZ) & $: 7.713059766$ \\
\hline Aeps1 1 & & $: 2.536$ \\
\hline |Aeps1 1 & (GZ) & $: 2.536843489960750 e+00$ \\
\hline Afinite & & $: 5.9$ \\
\hline $\mid$ Afinite $\mid$ & (GZ) & $: 5.933610502945470 \mathrm{e}+00$ \\
\hline Helicitie & $s:+-$ & +-+-+ \\
\hline tree & & 1.1018656 \\
\hline tree & (GZ) & : $1.101865680944420 \mathrm{e}-01$ \\
\hline |Aeps2 | & & : $7.713059766609913 \mathrm{e}-01$ \\
\hline |Aeps2 & (GZ) & $: 7.713059766610950 \mathrm{e}-01$ \\
\hline Aeps1 1 & & $: 2.536843489960436 \mathrm{e}+00$ \\
\hline Aeps1 1 & (GZ) & $: 2.536843489960750 \mathrm{e}+00$ \\
\hline Afinite & & : $6.042012410247624 \mathrm{e}+00$ \\
\hline |Afinite| & (GZ) & $: 6.042012409916140 \mathrm{e}+00$ \\
\hline
\end{tabular}


The lines marked with (GZ) correspond to the results as taken from Ref. [22].

\section{A.2 Comparison with Giele, Kunszt, Melnikov}

Running the sample program with option --GKMcheck should produce results similar to what is listed below:

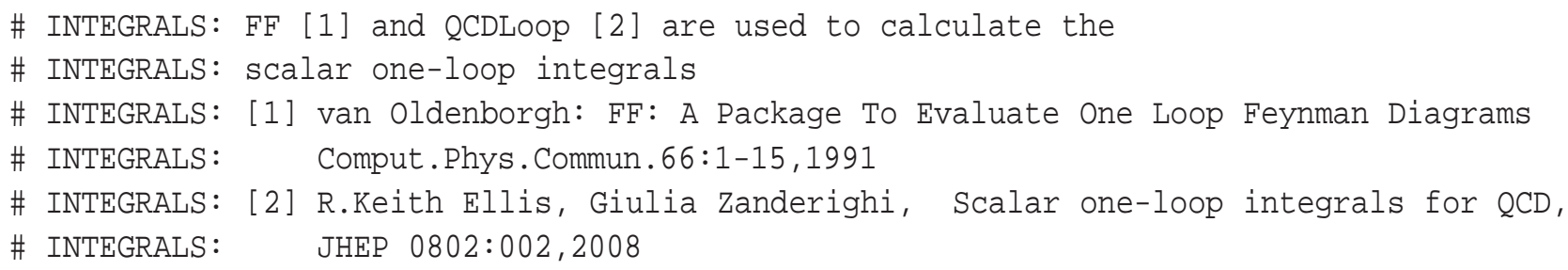




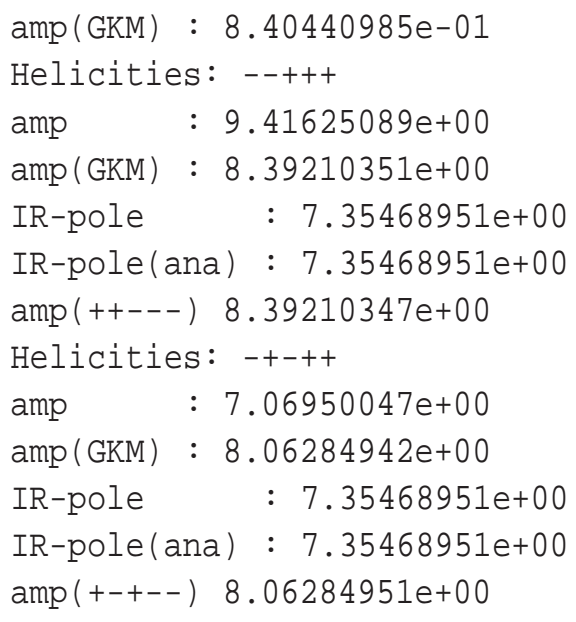


IR-pole (ana) : $2.03178718 e+00$

Helicities: -+-+-+

amp : $1.48181613 e+01$

amp (GKM) : $1.48181614 \mathrm{e}+01$

IR-pole : $2.03178718 \mathrm{e}+00$

IR-pole (ana) : $2.03178718 \mathrm{e}+00$

all checks with IR/UV poles and JHEP 0806:038,2008 passed.

\# Time used for this run: $4.00020000 \mathrm{e}-02$

We note that for the helicity configurations --+++ and -+-++ we disagree with Ref. [17].

However, we observed that switching to ++--- and +-+-- we find agreement. 


\section{References}

[1] A. Bredenstein, A. Denner, S. Dittmaier, and S. Pozzorini, Phys.Rev.Lett. 103, 012002 (2009), arXiv:arXiv:0905.0110.

[2] S. Dittmaier, S. Kallweit, and P. Uwer, Phys.Rev.Lett. 100, 062003 (2008), arXiv:arXiv:0710.1577.

[3] S. Dittmaier, P. Uwer, and S. Weinzierl, Phys.Rev.Lett. 98, 262002 (2007), arXiv:hep-ph/0703120.

[4] SM and NLO Multileg Working Group, J. Andersen et al., (2010), arXiv:1003.1241.

[5] Z. Bern, L. J. Dixon, D. C. Dunbar, and D. A. Kosower, Nucl. Phys. B425, 217 (1994), arXiv:hep$\mathrm{ph} / 9403226$.

[6] Z. Bern, L. J. Dixon, D. C. Dunbar, and D. A. Kosower, Nucl. Phys. B435, 59 (1995), arXiv:hep$\mathrm{ph} / 9409265$.

[7] R. Britto, F. Cachazo, and B. Feng, Nucl. Phys. B725, 275 (2005), arXiv:hep-th/0412103.

[8] G. Ossola, C. G. Papadopoulos, and R. Pittau, Nucl. Phys. B763, 147 (2007), arXiv:hep-ph/0609007.

[9] D. Forde, Phys. Rev. D75, 125019 (2007), arXiv:0704.1835.

[10] Z. Bern, L. J. Dixon, and D. A. Kosower, Phys.Rev. D71, 105013 (2005), arXiv:hep-th/0501240.

[11] Z. Bern, L. J. Dixon, and D. A. Kosower, Phys.Rev. D72, 125003 (2005), arXiv:hep-ph/0505055.

[12] Z. Bern, L. J. Dixon, and D. A. Kosower, Phys.Rev. D73, 065013 (2006), arXiv:hep-ph/0507005.

[13] C. F. Berger, Z. Bern, L. J. Dixon, D. Forde, and D. A. Kosower, Phys.Rev. D74, 036009 (2006), arXiv:hep$\mathrm{ph} / 0604195$.

[14] C. F. Berger, Z. Bern, L. J. Dixon, D. Forde, and D. A. Kosower, Phys.Rev. D75, 016006 (2007), arXiv:hep$\mathrm{ph} / 0607014$.

[15] C. Anastasiou, R. Britto, B. Feng, Z. Kunszt, and P. Mastrolia, Phys. Lett. B645, 213 (2007), arXiv:hep$\mathrm{ph} / 0609191$.

[16] C. Anastasiou, R. Britto, B. Feng, Z. Kunszt, and P. Mastrolia, JHEP 03, 111 (2007), arXiv:hep$\mathrm{ph} / 0612277$.

[17] W. T. Giele, Z. Kunszt, and K. Melnikov, JHEP 04, 049 (2008), arXiv:0801.2237.

[18] G. Ossola, C. G. Papadopoulos, and R. Pittau, JHEP 05, 004 (2008), arXiv:0802.1876.

[19] S. D. Badger, JHEP 01, 049 (2009), arXiv:0806.4600.

[20] R. K. Ellis, W. T. Giele, and Z. Kunszt, JHEP 03, 003 (2008), arXiv:0708.2398.

[21] C. F. Berger et al., Phys. Rev. D78, 036003 (2008), arXiv:0803.4180.

[22] W. Giele and G. Zanderighi, JHEP 0806, 038 (2008), arXiv:arXiv:0805.2152.

[23] W. Giele, Z. Kunszt, and J. Winter, (2009), arXiv:0911.1962.

[24] A. Lazopoulos, (2008), arXiv:arXiv:0812.2998.

[25] A. Lazopoulos, (2009), arXiv:arXiv:0911.5241.

[26] C. F. Berger et al., Phys. Rev. D80, 074036 (2009), arXiv:0907.1984.

[27] C. F. Berger et al., Phys. Rev. Lett. 102, 222001 (2009), arXiv:0902.2760.

[28] C. F. Berger et al., (2010), arXiv:1004.1659.

[29] C. Berger et al., (2010), arXiv:arXiv:1009.2338, * Temporary entry *.

[30] T. Melia, K. Melnikov, R. Rontsch, and G. Zanderighi, (2010), arXiv:arXiv:1007.5313.

[31] K. Melnikov and M. Schulze, Nucl.Phys. B840, 129 (2010), arXiv:arXiv:1004.3284.

[32] K. Melnikov and G. Zanderighi, Phys.Rev. D81, 074025 (2010), arXiv:arXiv:0910.3671.

[33] K. Melnikov and M. Schulze, JHEP 0908, 049 (2009), arXiv:arXiv:0907.3090.

[34] R. Ellis, K. Melnikov, and G. Zanderighi, JHEP 0904, 077 (2009), arXiv:arXiv:0901.4101.

[35] G. Bevilacqua, M. Czakon, C. Papadopoulos, and M. Worek, Phys.Rev.Lett. 104, 162002 (2010), arXiv:arXiv:1002.4009. 
[36] G. Bevilacqua, M. Czakon, C. Papadopoulos, R. Pittau, and M. Worek, JHEP 0909, 109 (2009), arXiv:arXiv:0907.4723.

[37] G. Ossola, C. G. Papadopoulos, and R. Pittau, JHEP 03, 042 (2008), arXiv:0711.3596.

[38] P. Mastrolia, G. Ossola, T. Reiter, and F. Tramontano, JHEP 1008, 080 (2010), arXiv:arXiv:1006.0710.

[39] G. 't Hooft and M. Veltman, Nucl.Phys. B153, 365 (1979).

[40] A. Denner, U. Nierste, and R. Scharf, Nucl.Phys. B367, 637 (1991), Dedicated to M. Veltman on occasion of his 60 th birthday.

[41] Z. Bern, L. J. Dixon, and D. A. Kosower, Nucl.Phys. B412, 751 (1994), arXiv:hep-ph/9306240.

[42] R. K. Ellis and G. Zanderighi, JHEP 02, 002 (2008), arXiv:0712.1851.

[43] G. van Oldenborgh and J. Vermaseren, Z.Phys. C46, 425 (1990).

[44] R. K. Ellis, W. T. Giele, Z. Kunszt, K. Melnikov, and G. Zanderighi, JHEP 01, 012 (2009), arXiv:0810.2762.

[45] T. Hahn and M. Perez-Victoria, Comput.Phys.Commun. 118, 153 (1999), arXiv:hep-ph/9807565.

[46] A. van Hameren, (2010), arXiv:arXiv:1007.4716.

[47] C. F. Berger and D. Forde, Ann.Rev.Nucl.Part.Sci. (2009), arXiv:arXiv:0912.3534.

[48] W. Giele and E. Glover, Phys.Rev. D46, 1980 (1992).

[49] F. A. Berends and W. Giele, Nucl.Phys. B306, 759 (1988).

[50] W. L. van Neerven and J. A. M. Vermaseren, Phys. Lett. B137, 241 (1984).

[51] G. Ossola, C. G. Papadopoulos, and R. Pittau, JHEP 07, 085 (2007), arXiv:0704.1271.

[52] F. del Aguila and R. Pittau, JHEP 07, 017 (2004), arXiv:hep-ph/0404120.

[53] Z. Bern, L. J. Dixon, D. C. Dunbar, and D. A. Kosower, Phys. Lett. B394, 105 (1997), arXiv:hepth/9611127.

[54] Y. Hida, X. S. Li, and D. H. Bailey, Library for Double-Double and Quad-Double Arithmetic, http://crd.lbl.gov/Ŏ2dcdhbailey/mpdist, report LBNL-46996, 2008.

[55] G. Mahlon, Phys.Rev. D49, 4438 (1994), arXiv:hep-ph/9312276.

[56] D. Forde and D. A. Kosower, Phys.Rev. D73, 061701 (2006), arXiv:hep-ph/0509358. 